On the dispersionless Kadomtsev-Petviashvili equation with arbitrary nonlinearity and dimensionality: exact solutions, longtime asymptotics of the Cauchy problem, wave breaking and shocks

This content has been downloaded from IOPscience. Please scroll down to see the full text.

2016 J. Phys. A: Math. Theor. 49405203

(http://iopscience.iop.org/1751-8121/49/40/405203)

View the table of contents for this issue, or go to the journal homepage for more

Download details:

IP Address: 141.108.19.33

This content was downloaded on 20/09/2016 at 14:29

Please note that terms and conditions apply.

You may also be interested in:

On the solutions of the dKP equation: the nonlinear Riemann Hilbert problem, longtime behaviour, implicit solutions and wave breaking

S V Manakov and P M Santini

On the dispersionless Kadomtsev--Petviashvili equation in $n+1$ dimensions: exact solutions, the

Cauchy problem for small initial data and wave breaking

S V Manakov and P M Santini

Shock formation in the dispersionless Kadomtsev-Petviashvili equation

T Grava, C Klein and J Eggers

Recovery of singularities in a fourth-order operator on the line from limited data

Teemu Tyni, Markus Harju and Valery Serov

On the reconstruction of boundary impedance of heat conduction system from nonlocal measurement

Jijun Liu and Yuchan Wang

Integrable structures in quantum field theory

Stefano Negro 


\title{
On the dispersionless Kadomtsev- Petviashvili equation with arbitrary nonlinearity and dimensionality: exact solutions, longtime asymptotics of the Cauchy problem, wave breaking and shocks
}

\author{
F Santucci ${ }^{1}$ and $\mathbf{P}$ M Santini ${ }^{2,3}$ \\ ${ }^{1}$ Istituto Superiore IIS G.Marconi, via Reno snc, I-04100 Latina, Italy \\ ${ }^{2}$ Dipartimento di Fisica, Università di Roma 'La Sapienza', and Istituto Nazionale di \\ Fisica Nucleare, Sezione di Roma 1 Piazz.le Aldo Moro 2, I-00185 Roma, Italy \\ E-mail: nadir17@libero.it and paolo.santini@roma1.infn.it
}

Received 21 December 2015, revised 23 May 2016

Accepted for publication 13 June 2016

Published 12 September 2016

\begin{abstract}
We study the generalization of the dispersionless Kadomtsev-Petviashvili (dKP) equation in $n+1$ dimensions and with nonlinearity of degree $m+1$, a model equation describing the propagation of weakly nonlinear, quasi onedimensional waves in the absence of dispersion and dissipation, and arising in several physical contexts, like acoustics, plasma physics, hydrodynamics and nonlinear optics. In $2+1$ dimensions and with quadratic nonlinearity, this equation is integrable through a novel inverse scattering transform, and it has been recently shown to be a prototype model equation in the description of the two-dimensional wave breaking of localized initial data. In higher dimensions and with higher nonlinearity, the generalized dKP equations are not integrable, but their invariance under motions on the paraboloid allows one to construct in this paper a family of exact solutions describing waves constant on their paraboloidal wave front and breaking simultaneously in all points of it, developing after breaking either multivaluedness or single-valued discontinuous profiles (shocks). Then such exact solutions are used to build the longtime behavior of the solutions of the Cauchy problem, for small and localized initial data, showing that wave breaking of small initial data takes place in the longtime regime if and only if $m(n-1) \leqslant 2$. Lastly, the analytic aspects of such wave breaking are investigated in detail in terms of the small initial data, in both cases in which the solution becomes multivalued after
\end{abstract}

3 Author to whom any correspondence should be addressed. 
breaking or it develops a shock. These results, contained in the 2012 master's thesis of one of the authors (FS) [1], generalize those obtained in [2] for the $\mathrm{dKP}$ equation in $n+1$ dimensions with quadratic nonlinearity, and are obtained following the same strategy.

Keywords: generalized dKP equations, exact solutions, small data Cauchy problem

(Some figures may appear in colour only in the online journal)

\section{Introduction}

In this paper we investigate the following generalized dispersionless Kadomtsev-Petviashvili (dKP) equation

$$
\begin{aligned}
& \left(u_{t}+u^{m} u_{x}\right)_{x}+\triangle_{\perp} u=0, \quad u=u(x, \vec{y}, t) \in \mathbb{R}, \\
& \triangle_{\perp}:=\sum_{i=1}^{n-1} \partial_{y_{i}}^{2}, \quad \vec{y}=\left(y_{1}, \cdots, y_{n-1}\right) \in \mathbb{R}^{n-1}, \quad x, t \in \mathbb{R},
\end{aligned}
$$

in $(n+1)$ dimensions, where $(m+1)$ is the degree of nonlinearity, and $m, n \in \mathbb{N}^{+}$. Hereafter, we refer to (1.1) as the $\operatorname{dKP}(m, n)$ equation. Equations (1.1) contain, as particular cases, the integrable (through the method of characteristics) Riemann equation [3]

$$
u_{t}+u^{m} u_{x}=0
$$

for $n=1$, the integrable dKP equation [4-7]

$$
\left(u_{t}+u u_{x}\right)_{x}+u_{y y}=0
$$

for $(m, n)=(1,2)$, the nonintegrable Khokhlov-Zabolotskaya (KZ) equation [6]

$$
\left(u_{t}+u u_{x}\right)_{x}+u_{y_{1} y_{1}}+u_{y_{2} y_{2}}=0
$$

for $(m, n)=(1,3)$, and the nonintegrable modified dKP (mdKP) equation [8]

$$
\left(u_{t}+u^{2} u_{x}\right)_{x}+u_{y y}=0
$$

for $(m, n)=(2,2)$.

Equation (1.1) describes weakly nonlinear and quasi one-dimensional waves, with negligible dissipation and dispersion, if the linear approximation of the original theory is given by the $(n+1)$-dimensional wave equation, at least in some limiting cases like longwave approximation [2]. In equation (1.1) there are two competing terms: the nonlinear term $u^{m} u_{x}$, responsible for the steepening of the profile, and the $\Delta_{\perp} u$ term, describing diffraction in the transversal $(n-1)$-dimensional hyperplane (reminiscence, through the multiscale expansion leading to (1.1), of the diffraction described by the wave operator); therefore diffraction increases as $n$ increases [2]. The degree $(m+1)$ of nonlinearity originates from expanding the nonlinear terms of the original PDEs in the power series, when the first $m$ powers of the expansion are absent due, usually, to some symmetry of the problem. In this sense, the degree of universality of (1.1) decreases as the degree of nonlinearity grows (see section 2). $\operatorname{dKP}(1, n), n=2,3$ are relevant in acoustics [2, 4-6], and also in plasma physics $[1,2,7]$ in long-wave approximation; $\operatorname{dKP}(1,2)$ in hydrodynamics, again in longwave approximation $[9,10] ; \operatorname{dKP}(1,3)$ and $\operatorname{dKP}(2,3)$ in nonlinear optics $[1]$; and $\operatorname{dKP}(2,2)$ in the study of sound waves in antiferromagnets [11]. 
We remark that equation (1.1) arises as the $x$-dispersionless limit of the natural generalization in $n+1$ dimensions and with nonlinearity of degree $m+1$ :

$$
\left(u_{t}+u_{x x x}+u^{m} u_{x}\right)_{x} \pm \triangle_{\perp} u=0, u=u(x, \vec{y}, t) \in \mathbb{R}
$$

of the $2+1$ dimensional Kadomtsev-Petviashvili equation [7]

$$
\left(u_{t}+u_{x x x}+u u_{x}\right)_{x} \pm u_{y y}=0, u=u(x, t) \in \mathbb{R} .
$$

This equation is integrable by the classical inverse scattering transform (IST) [12-15] (see also $[9,10,16])$ and the corresponding inverse problem has been used to construct the longtime behavior of its solutions [17-20].

Apart from the Riemann equation (1.2), integrable by the method of characteristics, in the family of equations (1.1) only the dKP equation (1.3) is integrable through a novel IST for integrable dispersionless PDEs [21-23], recently made rigorous in [24] on the example of the Pavlov equation [25]. This IST allows one to show, in particular, that solutions $u(x, y, t)$ of dKP depend on $x$ through the combination $x-2 u t$ [26]; i.e., these solutions can be written in the characteristic form [26, 27]

$$
u=F(\zeta, y, t), \quad \zeta=x-2 F(\zeta, y, t) t,
$$

in analogy with the case of the Riemann equation (1.2), for which the dependence of the solution $u(x, t)$ on $x$ is through the combination $x-u^{m} t$. For this reason, the IST for dKP can be viewed as a significant generalization of the method of characteristics. Formulation (1.8) has allowed one to study in an analytically explicit way the interesting features of the gradient catastrophe of two-dimensional waves at finite time [26, 27] and in the longtime regime [26] in terms of the initial data.

Other examples of $\operatorname{dKP}(m, n)$ equations are not integrable; therefore the possibility of investigating a generic wave breaking through equations like (1.8) and the precise form that these equations should take are, in our opinion, challenging open problems; in addition, blowup of the solutions is expected for sufficiently large $m$ [8] to complicate the picture. In recent papers [28] and [29], f.i., the formal dependences $x-u t$ and $x-u^{m} t$, respectively, motivated by the $y$ independent limit, were used to study the breaking features of $\operatorname{dKP}(1,2)$ and $\mathrm{dKP}(m, 2)$, respectively, together with its dissipative and dispersive shock formations, respectively.

In our paper we shed some light on the problem of finding a convenient characteristic form of the type (1.8) for the study of the wave breaking of $\operatorname{dKP}(m, n)$ solutions, (i) from the construction of a family of exact solutions of $\operatorname{dKP}(m, n)$ exhibiting wave breaking, and (ii) from the construction of the longtime behavior of solutions of the Cauchy problem of $\mathrm{dKP}(m, n)$, for small initial data.

Indeed, after showing in section 2 the universality of (1.1) starting from a family of nonlinear wave equations, in section 3 we use the invariance of the $\operatorname{dKP}(m, n)$ equations under motions on the paraboloid, to construct a family of exact solutions involving an arbitrary function of one variable, and describing waves constant on their paraboloidal wave front, breaking simultaneously in all points of it, and developing, after breaking, either multivalued overturning profiles or single-valued discontinuous profiles (shocks). Then we use in section 4 such solutions to build a uniform approximation of the solution of the Cauchy problem, in the longtime regime and for small and localized initial data, showing that such small and localized data evolving according to the $\operatorname{dKP}(m, n)$ equation break, in the longtime regime, iff $m(n-1) \leqslant 2$. In 5 we study the analytic aspects of such wave breaking, given explicitly in terms of the initial data, providing, in particular, a description of the overturning profile and of the development of a shock immediately after breaking; we concentrate, in 
particular, on the mdKP (1.5) case and on its comparison with the already known dKP (1.3) case. The results of this paper, contained in the 2012 master's thesis of one of the authors (FS) [1], generalize analogous ones for the $\operatorname{dKP}(1, n)$ equation in [2], and are obtained following the same strategy.

We end this introduction considering the following consequence of these results: for the family of exact solutions of $\operatorname{dKP}(m, n)$ and for the longtime asymptotics of the solutions of the small data Cauchy problem, the dependence of the solutions $u(x, \vec{y}, t)$ on $x$ is, f.i., through the combinations $x-c_{m, n}^{-1} u^{m} t$, if $c_{m, n}>0$, and $x-u^{m} t \log t$, if $c_{m, n}=0$, where

$$
c_{m, n}=1-\frac{m(n-1)}{2} .
$$

Therefore this dependence involves in a simple way the degree $m+1$ of the nonlinearity and the dimensionality $n+1$ of the problem through the coefficient $c_{m, n}$, and it is consistent with the case of the Riemann equation (1.2), for which $c_{m, 1}=1$, and with the dKP equation (1.3), for which $c_{1,2}=1 / 2$ (confirming the apparently mysterious factor 2 in (1.8)); it is not consistent, in contrast, with the formal dependence $x-u^{m} t$ used in [29].

In analogy with the dKP case, the results of our paper seem to suggest the following conjecture (admittedly a weak conjecture, due to the absence, in the generic case, of an IST generalizing the method of characteristics).

Conjecture. The solutions of the $\operatorname{dKP}(m, n)$ equation around breaking, for $c_{m, n} \geqslant 0$, are described by the following characteristic formulae:

$$
\begin{aligned}
& u=G(\zeta, y, t), \\
& \zeta= \begin{cases}x-c_{m, n}^{-1} G^{m}(\zeta, \vec{y}, t) t, & \text { if } c_{m, n}>0, \\
x-G^{m}(\zeta, \vec{y}, t) t \log t, & \text { if } c_{m, n}=0 .\end{cases}
\end{aligned}
$$

This paper is dedicated to the memory of S. V. Manakov.

\section{Universality and applicability of $\operatorname{dKP}(m, n)$}

The universality (and therefore the applicability) of (1.1), through a multiscale expansion, is well described by the following simple model: the family of nonlinear wave equations

$$
(f(w))_{T T}=\Delta w, \quad \Delta=\sum_{i=1}^{n} \partial_{X_{i}}^{2}, \quad w=w(\vec{X}, T) .
$$

For small amplitudes: $w \rightarrow \epsilon w, 0<\epsilon \ll 1$, we substitute in (2.1) the nonlinear term by its Taylor expansion

$$
f(\epsilon w)=f(0)+f^{\prime}(0) \epsilon w+\frac{1}{2} f^{\prime \prime}(0) \epsilon^{2} w^{2}+\cdots .
$$

obtaining, at $O(\epsilon)$, the wave equation

$$
w_{T T}=c^{2} \triangle w, \quad c=1 / \sqrt{f^{\prime}(0)},
$$

where $f^{\prime}(0)$ is assumed to be positive.

If the waves are quasi one-dimensional and we choose $X_{1}$ as the direction of propagation, the wave vector in the transversal directions is small: $\vec{k}_{\perp}=\epsilon^{\alpha} \vec{k}_{\perp}$, where $\vec{k}_{\perp}$ is the transversal wave vector and $\alpha>0$ has to be fixed. Then the dispersion relation becomes [2] 


$$
\omega=c \sqrt{k_{1}^{2}+\vec{k}_{\perp}^{2}}=c k_{1} \sqrt{1+\epsilon^{2 \alpha} \frac{\vec{k}_{\perp}^{2}}{k_{1}^{2}}} \simeq c k_{1}\left(1+\epsilon^{2 \alpha} \frac{\vec{k}_{\perp}^{2}}{2 k_{1}^{2}}\right),
$$

and the phase of a monochromatic wave reads

$$
\vec{k} \cdot \vec{X}-\omega T=k_{1}\left(X_{1}-c T\right)+\epsilon^{\alpha} \vec{k}_{\perp} \cdot \vec{X}_{\perp}-\frac{c \epsilon^{2 \alpha}}{2} \frac{\vec{\kappa}_{\perp}^{2}}{k_{1}^{2}} T,
$$

motivating the introduction of the new variables

$$
\left\{\begin{array}{l}
x=X_{1}-c T, \\
\vec{y}=\epsilon^{\alpha} \vec{X}_{\perp}, \quad y_{i}=X_{i+1}, \quad i=1, \ldots, n-1, \\
t=\epsilon^{2 \alpha} \frac{c}{2} T .
\end{array}\right.
$$

Rewriting (2.1) in the new variables and imposing $\alpha=1 / 2$ to get the maximal balance, one obtains, at $O\left(\epsilon^{2}\right)$, the $\mathrm{dKP}(1, n)$ equation

$$
\left(u_{t}+u u_{x}\right)_{x}+\triangle_{\perp} u=0,
$$

where $u=-\frac{c^{2}}{2} f^{\prime \prime}(0) w$, and $\Delta_{\perp}$ is the transversal Laplacian given in (1.1b).

If the term $f^{\prime \prime}(0)$ vanishes, the maximal balance must involve the cubic term; if also $f^{\prime \prime \prime}(0)=0$, the maximal balance must involve the quartic term, and so on. In the very special case in which $f^{(l)}(0)=0, l=2, \ldots m$, and $f^{(m+1)}(0) \neq 0$, the maximal balance must involve the term of order $m+1$, and is achieved for $\alpha=m / 2$ at the first nontrivial order $O\left(\epsilon^{m+1}\right)$, obtaining the $\operatorname{dKP}(m, n)$ equation (1.1), with $u=C^{1 / m} w$ and

$$
C:=-\frac{c^{2} f^{(m+1)}(0)}{m !},
$$

if $m$ is odd, and

$$
\left(u_{t}-\operatorname{sgn}\left(f^{(m+1)}(0)\right) u^{m} u_{x}\right)_{x}+\triangle_{\perp} u=0,
$$

with $u=|C|^{1 / m} w$, if $m$ is even.

The above considerations explain well why equations (1.1) are less and less universal and applicable as $m$ increases.

\section{Exact solutions of $\operatorname{dKP}(m, n)$}

It was observed in [2] that $\operatorname{dKP}(1, n)$ is invariant under the following Lie symmetry group of transformations:

$$
\left\{\begin{array}{l}
\tilde{x}=x+\sum_{i=1}^{n-1}\left(\delta_{i} y_{i}-\delta_{i}^{2} t\right) \\
\tilde{y}_{j}=y_{j}-2 \delta_{j} t, \quad j=1, \cdots, n-1, \\
\tilde{t}=t
\end{array}\right.
$$

where $\delta_{j}, j=1, \ldots, n-1$, are the arbitrary parameters of the group, leaving invariant the paraboloid

$$
\xi=x+\frac{1}{4 t} \sum_{i=1}^{n-1} y_{i}^{2}
$$


Such a symmetry was used to construct a family of exact and implicit solutions of $\operatorname{dKP}(1, n)$ exhibiting wave breaking and playing a relevant role in the longtime regime of the small data Cauchy problem for $\mathrm{dKP}(1, n)[2]$.

Since $\operatorname{dKP}(m, n)$ is also invariant under the transformation (3.1), following the same strategy as in [2], such a symmetry will be used in this section to construct a family of exact and implicit solutions of $\operatorname{dKP}(m, n)$. In this paper we also add, to the exact solutions exhibiting a gradient catastrophe and multivaluedness after breaking, the exact weak solutions of $\mathrm{dKP}(m, n)$ developing, after breaking, single-valued discontinuous shocks of a dissipative nature.

Therefore we look for localized solutions of (1.1) in the form

$$
u=v(\xi, t), \quad \xi=x+\frac{1}{4 t} \sum_{i=1}^{n-1} y_{i}^{2},
$$

reducing $(1.1)$ to the $(1+1)$-dimensional PDE

$$
v_{t}+v^{m} v_{\xi}+\frac{n-1}{2 t} v=0 .
$$

The following change of variables

$$
v(\xi, t)=t^{-\frac{n-1}{2}} q(\xi, \tau)
$$

where

$$
\tau(t)=\left\{\begin{array}{ll}
\frac{1}{c_{m, n}} t^{c_{m, n}}+\alpha, & \text { if } c_{m, n} \neq 0 \\
\ln t+\beta, & \text { if } c_{m, n}=0
\end{array},\right.
$$

$\alpha, \beta$ are real constants and the coefficient $c_{m, n}$ is defined in (6.1), transforms (3.4) into the Riemann equation (1.2) in the variables $(\xi, \tau)$

$$
q_{\tau}+q^{m} q_{\xi}=0
$$

We recall [3] that (3.7) has the general implicit solution

$$
q=A(\zeta), \quad \zeta=\xi-A^{m}(\zeta) \tau \Leftrightarrow q=A\left(\xi-q^{m} \tau\right),
$$

where $A$ is an arbitrary differentiable function of one argument. If, in particular, $A$ describes a localized positive hump, the solution breaks first at $\tau=\tau_{\mathrm{b}}$, on the characteristic $\zeta_{\mathrm{b}}$, where

$$
\tau_{\mathrm{b}}=\min \zeta\left(-\frac{1}{m A^{m-1}(\zeta) A^{\prime}(\zeta)}\right)=-\frac{1}{m A^{m-1}\left(\zeta_{\mathrm{b}}\right) A^{\prime}\left(\zeta_{\mathrm{b}}\right)}>0 .
$$

For $\tau>\tau_{\mathrm{b}}$, the solution becomes multivalued and not acceptable in many physical contexts. Alternatively, for $\tau>\tau_{\mathrm{b}}$, the regular multivalued solution can be replaced by a weak solution, a single-valued shock of a dissipative nature, whose wave front discontinuity $s(\tau)$ is described by equations [3]

$$
\begin{aligned}
\frac{\mathrm{d} s}{\mathrm{~d} \tau} & =\frac{1}{m+1} \frac{A^{m+1}\left(\zeta_{1}\right)-A^{m+1}\left(\zeta_{2}\right)}{A\left(\zeta_{1}\right)-A\left(\zeta_{2}\right)}, \\
s & =\zeta_{1}+A^{m}\left(\zeta_{1}\right) \tau=\zeta_{2}+A^{m}\left(\zeta_{2}\right) \tau,
\end{aligned}
$$

with the initial conditions

$$
s\left(\tau_{\mathrm{b}}\right)=\xi_{\mathrm{b}}, \quad \zeta_{1}\left(\tau_{\mathrm{b}}\right)=\zeta_{2}\left(\tau_{\mathrm{b}}\right)=\zeta_{\mathrm{b}}
$$



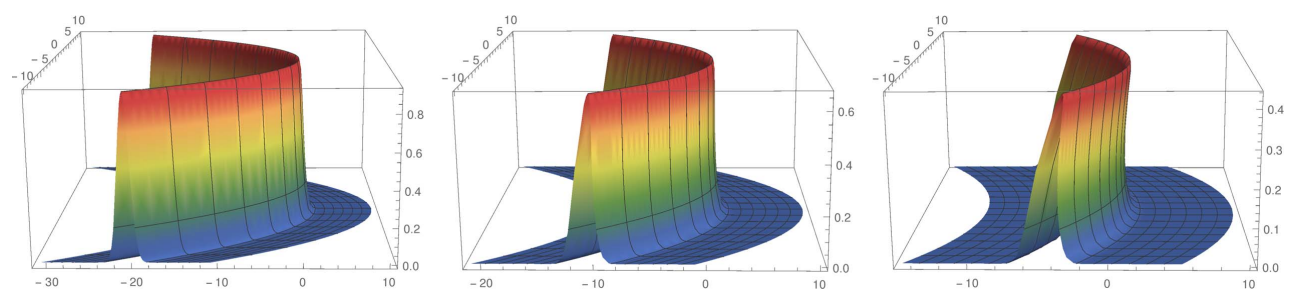

Figure 1. Plotting of the exact solution of the mdKP equation (1.5) $\left(c_{2,2}=0\right)$, before, at and after breaking. Here we have chosen $A(x)=\mathrm{e}^{-x^{2}} ;$ consequently: $\zeta_{\mathrm{b}}=1 / 2$, $\tau_{\mathrm{b}}=\sqrt{\mathrm{e}} / 2, \quad t_{\mathrm{b}}=\mathrm{e}^{\sqrt{\mathrm{e}} / 2}$.

Behind and ahead the shock, the solution is given by

$$
q= \begin{cases}q_{2}=A\left(\zeta_{2}\right), & \text { if } \xi<s(\tau), \\ q_{1}=A\left(\zeta_{1}\right), & \text { if } \xi>s(\tau),\end{cases}
$$

where $q_{2}$ and $q_{1}$ are respectively the maximum and the minimum among the (three, in the case of a single hump) branches of the implicit equations (3.8).

We remark that $\tau(t)$ in (3.6) is a monotonically increasing function of $t$; to have it positive, we choose the constants $\alpha, \beta$ and the $t$-intervals as follows:

$$
\tau(t)= \begin{cases}\frac{1}{c_{m, n}} t^{c_{m, n}, t>0,} & \text { if } c_{m, n}>0, \\ \ln t, t>1, & \text { if } c_{m, n}=0, \\ \frac{1}{\left|c_{m, n}\right|}\left(t_{0}^{-\left|c_{m, n}\right|}-t^{-\left|c_{m, n}\right|}\right), t>t_{0}, & \text { if } c_{m, n}<0,\end{cases}
$$

Recalling (3.3), (3.5), and (3.13), the exact solutions of $\operatorname{dKP}(m, n)$ in the original variables corresponding to (3.8) read

$$
u= \begin{cases}t^{-\frac{n-1}{2}} A\left(x+\frac{1}{4 t} \sum_{i=1}^{n-1} y_{i}^{2}-\frac{1}{c_{m, n}} u^{m} t\right), t>0, & \text { if } c_{m, n}>0 \\ t^{-\frac{n-1}{2}} A\left(x+\frac{1}{4 t} \sum_{i=1}^{n-1} y_{i}^{2}-u^{m} t \ln t\right), t>1, & \text { if } c_{m, n}=0 \\ t^{-\frac{n-1}{2}} A\left(x+\frac{1}{4 t} \sum_{i=1}^{n-1} y_{i}^{2}-\frac{1}{\left|c_{m, n}\right|} u^{m} t\left(\left(\frac{t}{t_{0}}\right)^{\left|c_{m, n}\right|}-1\right)\right), t>t_{0}, & \text { if } c_{m, n}<0,\end{cases}
$$

where $c_{m, n}$ is defined in (6.1).

If, again, $A$ is a localized hump, (3.14) describes a wave constant on the paraboloidal wave front (3.2) and simultaneously breaking on it. More precisely, the solution breaks at time $t_{\mathrm{b}}$ defined by

$$
t_{\mathrm{b}}= \begin{cases}\left(c_{m, n} \tau_{\mathrm{b}}\right)^{1 / c_{m, n},}, & \text { if } c_{m, n}>0, \\ \mathrm{e}^{\tau_{\mathrm{b}},} & \text { if } c_{m, n}=0,\end{cases}
$$



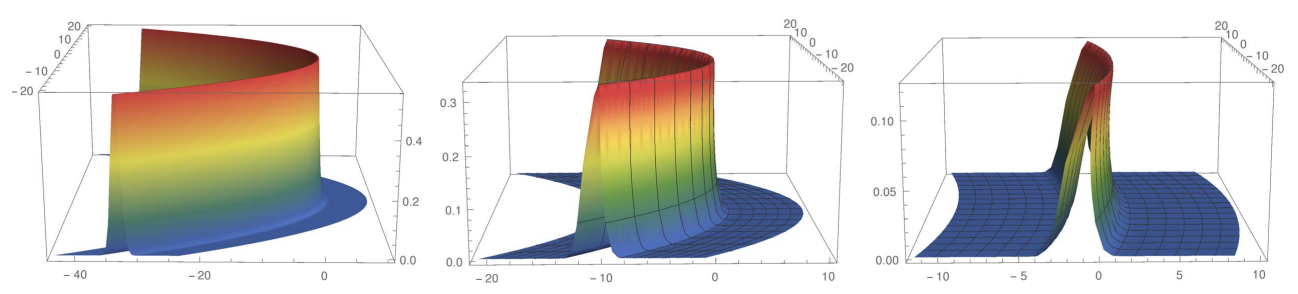

Figure 2. Plotting of the exact solution of the $\operatorname{dKP}(3,2)$ equation $\left(c_{3,2}<0\right)$ before, at and after breaking, and before freezing. Here we have chosen $A(x)=\mathrm{e}^{-x^{2}}$ and $t_{0}=2$; consequently: $\zeta_{\mathrm{b}}=1 / 2, \quad \tau_{\mathrm{b}}=\sqrt{\mathrm{e}} / 2$, and $t_{0}^{-\left|c_{m, n}\right|}>\tau_{\mathrm{b}}\left|c_{m, n}\right|$. Therefore the solution breaks, according to (3.17), at $t_{\mathrm{b}}=11.49$, before freezing.

on the paraboloid

$$
x+\frac{1}{4 t_{\mathrm{b}}} \sum_{i=1}^{n-1} y_{i}^{2}=\zeta_{\mathrm{b}}+A^{m}\left(\zeta_{\mathrm{b}}\right) \tau_{\mathrm{b}},
$$

where $\tau_{\mathrm{b}}, \zeta_{\mathrm{b}}$ are defined in (3.9) (see figure 1).

If $c_{m, n}<0, \tau(t) \rightarrow \tau(\infty) \equiv\left|c_{m, n}\right|^{-1} t_{0}^{-\left|c_{m, n}\right|}$ as $t \rightarrow \infty$, and the wave freezes asymptotically. If $t_{0}^{-\left|c_{m, n}\right|}>\tau_{\mathrm{b}}\left|c_{m, n}\right|$, then $\tau(\infty)>\tau_{\mathrm{b}}$, and the wave breaks before freezing at

$$
t_{\mathrm{b}}=\left(t_{0}^{-\left|c_{m, n}\right|}-\tau_{\mathrm{b}}\left|c_{m, n}\right|\right)^{-1 /\left|c_{m, n}\right|}
$$

on the paraboloid (3.16) (see figure 2). If, instead, $t_{0}^{-\left|c_{m, n}\right|}<\tau_{\mathrm{b}}\left|c_{m, n}\right|$, then $\tau(\infty)<\tau_{\mathrm{b}}$ and no breaking takes place before the wave freezes.

If $n=1, \forall m$, (3.14) reduces to the general solution $u=A\left(x-u^{m} t\right)$ of the Riemann equation (1.2). Subcases of the above solutions in the multidimensional context were obtained in [30] for the KZ equation (1.4), in [31] for the dKP equation (using its nonlinear RiemannHilbert inverse problem), and in [2] for the $\operatorname{dKP}(1, n)$ equations.

After breaking: $t>t_{\mathrm{b}}$, the regular multivalued solutions (3.14) can be replaced by the discontinuous single-valued solutions of $\operatorname{dKP}(m, n)$ obtained using (3.3), (3.5), and (3.13) in (3.10), (3.11), and (3.12). More precisely, for $t>t_{\mathrm{b}}$, where $t_{\mathrm{b}}$ is defined in (3.15) and (3.17), the $\operatorname{dKP}(m, n)$ solution described by (3.14) for $t<t_{\mathrm{b}}$ develops a shock on the parabola

$$
x+\frac{y^{2}}{4 t}=S(t), \quad S(t) \equiv s(\tau(t)),
$$

where $s(\tau)$ is characterized by equations (3.10) and (3.11), and $\tau(t)$ is defined in (3.13). Behind and ahead the shock, the solution is given by

$$
u= \begin{cases}u_{2}(x, \vec{y}, t), & \text { if } x+\frac{y^{4}}{4 t}<S(t), \\ u_{1}(x, \vec{y}, t), & \text { if } x+\frac{y^{4}}{4 t}>S(t),\end{cases}
$$

where $u_{2}$ and $u_{1}$ are respectively the maximum and the minimum among the (three, in the case of a localized bump) branches $u_{1,2}=t^{-(n-1) / 2} A\left(\zeta_{1,2}\right)$ of the implicit equations (3.14).

We end this section remarking that, as already mentioned in section 1 , in the above class of exact solutions of (1.1) the dependences of $u$ on $x$ through the combinations $x-c_{m, n}^{-1} u^{m} t$ (if $c_{m, n}>0$ ), $x-u^{m} t \log t$ (if $c_{m, n}=0$ ), and $x-\left|c_{m, n}\right|^{-1} u^{m} t\left[\left(t / t_{0}\right)^{\left|c_{m, n}\right|}-1\right]\left(\right.$ if $c_{m, n}<0$ ) are not consistent with the formal dependence $x-u^{m} t$ recently postulated to study the breaking features of $\operatorname{dKP}(m, 2)$ in [29]. 


\section{The Cauchy problem for small and localized initial data and longtime wave breaking}

In analogy with [2], now we show the relevance of the exact solutions of the previous section in the construction of the longtime behavior of solutions of the Cauchy problem of (1.1) for small and localized initial data, showing in particular that small and localized initial data evolving according to (1.1) break, in the longtime regime, only if $m(n-1) \leqslant 2\left(c_{m, n} \geqslant 0\right)$.

For small and localized initial data of the type

$$
u(x, \vec{y}, 0)=\epsilon u_{0}(x, \vec{y}), \quad 0<\epsilon \ll 1
$$

evolving according to equation (1.1), the behavior of the solution may be approximated by the solution of the linearized $\operatorname{dKP}(m, n)$ equation until nonlinearity becomes relevant.

For the $O(\epsilon)$ initial data, equations (3.7) and (3.9) imply that the breaking time is

$$
\tau_{\mathrm{b}}=O\left(\epsilon^{-m}\right) \gg 1
$$

so, the nonlinear regime for $\operatorname{dKP}(m, n)$ is characterized by the condition

$$
t=O\left(\tau^{-1}\left(\epsilon^{-m}\right)\right)= \begin{cases}O\left(\epsilon^{-\frac{m}{c_{m, n}}}\right), & c_{m, n}>0 \\ O\left(\mathrm{e}^{\epsilon^{-m}}\right), & c_{m, n}=0\end{cases}
$$

where $\tau^{-1}(\cdot)$ is the inverse of (3.6), while, for $c_{m, n}<0$, large values of $t$ are not compatible with (4.2), and a nonlinear regime never occurs.

So, for $t \ll \tau^{-1}\left(\epsilon^{-m}\right)$, the solution of the Cauchy problem is approximated by the solution of the linearized $\operatorname{dKP}(m, n)$ equation, while for $t \sim \tau^{-1}\left(\epsilon^{-m}\right)$, a matching will be made between the linearized solution and the exact solution (3.14).

In the linear regime, when $0<t \ll O\left(\tau^{-1}\left(\epsilon^{-m}\right)\right)$, the $\operatorname{dKP}(m, n)$ solution is well described by that of the linearized $\operatorname{dKP}(m, n)$, i.e.:

$$
u(x, \vec{y}, t) \sim \frac{\epsilon}{(2 \pi)^{n}} \int_{\mathbb{R}^{n}} \hat{u}_{0}\left(k_{1}, \vec{k}_{\perp}\right) \exp \left[l\left(k_{1} x+\vec{k}_{\perp} \cdot \vec{y}-\frac{k_{\perp}^{2}}{k_{1}} t\right)\right] \mathrm{d} k_{1} \mathrm{~d} \vec{k}_{\perp}
$$

where

$$
\hat{u}_{0}\left(k_{1}, \vec{k}_{\perp}\right)=\int_{\mathbb{R}^{n}} u_{0}(x, \vec{y}) \exp \left[-\imath\left(k_{1} x+\vec{k}_{\perp} \cdot \vec{y}\right)\right] \mathrm{d} x \mathrm{~d} \vec{y} .
$$

In the longtime regime

$$
1 \ll t \ll O\left(\tau^{-1}\left(\epsilon^{-m}\right)\right),
$$

the multiple integral can be evaluated by the stationary phase method, which gives [2]

$$
u(x, \vec{y}, t) \sim t^{-\frac{n-1}{2}} \epsilon G\left(x+\frac{1}{4 t} \sum_{i=1}^{n-1} y_{i}^{2}, \frac{\vec{y}}{2 t}\right),
$$

where

$G(\xi, \vec{\eta}):=\frac{1}{2^{n} \pi^{\frac{n+1}{2}}} \int_{\mathbb{R}} \mathrm{d} k_{1}\left|k_{1}\right|^{\frac{n-1}{2}} \hat{u}_{0}\left(k_{1}, \vec{\eta} k_{1}\right) \exp \left[\imath k_{1} \xi-\imath(n-1) \frac{\pi}{4} \operatorname{sgn}\left(k_{1}\right)\right]$.

in the space-time region

$$
(x-\xi) / t, y_{i} / t=O(1), \quad i=1, \ldots, n-1,
$$

on the paraboloid (3.2), and decays faster outside it. Therefore, small and localized initial data are set, in the longtime regime (4.5) governed by linear theory, on the paraboloid (3.2). 
When nonlinearity becomes relevant, i.e., when $t=O\left(\tau^{-1}\left(\epsilon^{-m}\right)\right)$, the asymptotic solution of $\operatorname{dKP}(m, n)$ is built matching the longtime solution (4.6), (4.7) of the linearized Cauchy problem with the exact solution (3.14). If, f.i., $c_{m, n}>0$, the first argument $\xi$ of function $G(\xi, \vec{\eta})$ defined in (4.7) is replaced by $\xi-c_{m, n}^{-1} u^{m} t$, or, equivalently, the arbitrary function $A(\zeta)$ in (3.8) acquires dependence on the second argument $\vec{\eta}=\vec{y} / 2 t$, and is identified with $G$. As a result of this matching, in the nonlinear regime (4.2), (4.3) the solution reads

$$
\begin{aligned}
& u(x, \vec{y}, t) \simeq u_{m, n}^{a s}(x, \vec{y}, t), \\
& u_{m, n}^{a s}(x, \vec{y}, t) \equiv\left\{\begin{array}{l}
t^{-\frac{n-1}{2}} \epsilon G\left(x+\frac{1}{4 t} \sum_{i=1}^{n-1} y_{i}^{2}-\frac{1}{c_{m, n}} u^{m} t, \frac{\vec{y}}{2 t}\right), \quad c_{m, n}>0, \\
t^{-\frac{n-1}{2}} \epsilon G\left(x+\frac{1}{4 t} \sum_{i=1}^{n-1} y_{i}^{2}-u^{m} t \ln t, \frac{\vec{y}}{2 t}\right), \quad c_{m, n}=0,
\end{array}\right.
\end{aligned}
$$

with $u^{m} t=O(1)$ for $c_{m, n}>0$, and $u^{m} t \log t=O(1)$ for $c_{m, n}=0$, becoming infinitesimal in the linear regime, as it has to be.

For $c_{m, n}<0$, the solution is described by its linear form (4.6); therefore wave breaking takes place for the particular values of $(m, n)$ described by the condition

$$
c_{m, n} \geqslant 0 \quad \Leftrightarrow \quad m(n-1) \leqslant 2 ;
$$

i.e., for

$$
\begin{array}{|l|l|l|}
\hline n=1 & n=2 & n=3 \\
\forall m & m=1,2 & m=1 \\
\hline
\end{array}
$$

For $n=1, \quad \forall m, u_{m, n}^{a s}$ reduces to the general solution of the Riemann equation (1.2) [3]. The cases $(m, n)=(2,1)$ and $(m, n)=(3,1)$, the dKP and the KZ equations, have been already investigated in [26] and [2] respectively; therefore, in the following section we mainly focus on the case $(m, n)=(2,2)$, corresponding to the nonintegrable mdKP equation (1.5) and to its asymptotic solution

$$
u \simeq u_{2,2}^{a s}(x, y, t)=\frac{1}{\sqrt{t}} \epsilon G\left(x+\frac{y^{2}}{4 t}-u^{2} t \ln t, \frac{y}{2 t}\right),
$$

comparing the results with the $\mathrm{dKP}$ case $(m, n)=(1,2)$ investigated in [26], with its asymptotic solution

$$
u \simeq u_{1,2}^{a s}(x, y, t)=\frac{1}{\sqrt{t}} \epsilon G\left(x+\frac{y^{2}}{4 t}-2 u t, \frac{y}{2 t}\right)
$$

Remark 1. The estimate of the first correction to the asymptotics (4.9) reads

$$
u=u_{n, m}^{a s}(x, \vec{y}, t)\left(1+O\left(t^{-1}\right)\right) \text {. }
$$

Indeed, if $c_{m, n}>0$, one has

$$
u \sim u_{n, m}^{a s}(x, \vec{y}, t)+\frac{\epsilon^{1+\frac{m}{c_{m, n}}}}{t^{\frac{n-1}{2}}} H\left(x+\frac{1}{4 t} \sum_{i=1}^{n-1} y_{i}^{2}, \frac{\vec{y}}{2 t}, \check{\tau}\right),
$$


where $\check{\tau}=\epsilon^{m} \tau$ and $H(\xi, \vec{\eta}, \check{\tau})$ is expressed in terms of $G(\zeta, \vec{\eta})$ ( $\zeta$ is defined in the second equation of (5.1)), through the PDE

$$
\left(\frac{H_{\check{\tau}}+G^{m} H_{\xi}}{1+m G^{m-1} G_{\zeta} \check{\tau}}\right)_{\xi}+\frac{1}{4\left(c_{m, n} \check{\tau}\right)^{1+c_{m, n}^{-1}}} \sum_{j=1}^{n-1}\left(\frac{G_{\eta_{j}}}{1+m G^{m-1} G_{\zeta} \check{\tau}}\right)_{\eta_{j}}=0
$$

Since, from (4.3), $\epsilon^{\frac{m}{c_{m, n}}}=O\left(t^{-1}\right)$, equation (4.15) yields (4.14). Similarly, if $c_{m, n}=0$,

$$
u \sim u_{n, m}^{a s}(x, \vec{y}, t)+\frac{\epsilon}{t^{\frac{n+1}{2}}} H\left(x+\frac{1}{4 t} \sum_{i=1}^{n-1} y_{i}^{2}, \frac{\vec{y}}{2 t}, \check{\tau}\right)
$$

where $H(\xi, \vec{\eta}, \check{\tau})$ now solves the PDE

$$
\left(\frac{H}{1+m G^{m-1} G_{\zeta} \check{\tau}}\right)_{\xi}=\frac{1}{4} \sum_{j=1}^{n-1}\left(\frac{G_{\eta_{j}}}{1+m G^{m-1} G_{\zeta} \check{\tau}}\right)_{\eta_{j}}
$$

Then equation (4.17) yields equation (4.14) too.

Remark 2. The leading term (4.9) of the solution of the Cauchy problem of (1.1), for small and localized initial data (4.1), combines in a simple way features of the linearized theory, described by equations (4.6) and (4.7), and of the exact lower-dimensional solutions (3.14) describing a wave constant on its paraboloidal wave front and simultaneously breaking on it. In particular, the dependence of (4.9) on the first argument, describing the wave breaking in the longitudinal direction, is the same as in the exact solutions (3.14), while the dependence on the remaining $n-1$ arguments, describing the localization properties of the wave front and, therefore, its multidimensionality, is the same as in the linearized theory (4.6), (4.7).

Remark 3. As is observed in [2], for a Gaussian initial condition

$$
u_{0}(x, y)=d \exp \left[-\frac{x^{2}+\vec{y}^{2}}{4}\right]
$$

where $d$ is constant, the asymptotic solution can be written in terms of special functions, obtaining for $G(\xi, \vec{\eta})$ :

$$
\begin{aligned}
G(\xi, \vec{\eta}) & \\
= & \frac{d}{\sqrt{\pi}} \frac{1}{\left(1+\vec{\eta}^{2}\right)^{\frac{n+1}{4}}}\left[\cos \left(\frac{\pi}{4}(n-1)\right) \Gamma\left(\frac{n+1}{4}\right){ }_{1} F_{1}\left(\frac{n+1}{4}, \frac{1}{2},-\frac{Y^{2}}{4}\right)\right. \\
& \left.+\sin \left(\frac{\pi}{4}(n-1)\right) \Gamma\left(\frac{n+3}{4}\right) Y_{1} F_{1}\left(\frac{n+3}{4}, \frac{3}{2},-\frac{Y^{2}}{4}\right)\right]
\end{aligned}
$$

where

$$
Y=\frac{\xi}{\sqrt{1+\vec{\eta}^{2}}}
$$

$\Gamma$ is the Euler gamma function and ${ }_{1} F_{1}$ is the Kummer confluent hypergeometric function $[32,33]$. For $n=2$ and $d=\sqrt{2 \pi}$, it yields, respectively: 


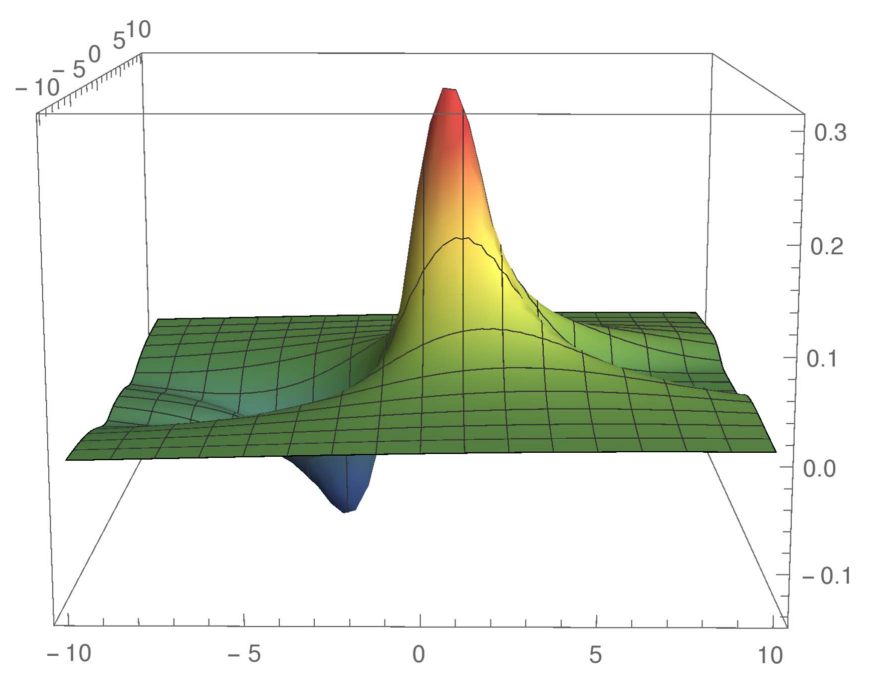

Figure 3. Plotting of function (4.22).

$$
\begin{aligned}
& G(\xi, \eta) \\
& \quad=\frac{1}{\left(1+\eta^{2}\right)^{\frac{3}{4}}}\left[\Gamma\left(\frac{3}{4}\right){ }_{1} F_{1}\left(\frac{3}{4}, \frac{1}{2},-\frac{Y^{2}}{4}\right)+Y \Gamma\left(\frac{5}{4}\right){ }_{1} F_{1}\left(\frac{5}{4}, \frac{3}{2},-\frac{Y^{2}}{4}\right)\right],
\end{aligned}
$$

shown in figure 3.

Remark 4. The fact that small data break only when inequality (4.10) is satisfied is expected from physical considerations. Indeed, as already mentioned in the introduction, in equation (1.1) there are two competing terms: the nonlinear term $u^{m} u_{x}$, responsible for the steepening of the profile, and the $\triangle_{\perp} u$ term, describing diffraction in the transversal $(n-1)$-dimensional hyperplane (reminiscence, through the multiscale expansion leading to (1.1), of the wave operator); therefore diffraction increases as $(n-1)$ increases [2]. For small initial data, the $u^{m} u_{x}$ term initially becomes smaller and smaller increasing $m$, and the solution evolves in a linear way for longer and longer time, diffracting transversally through the $(n-1)$ diffraction channels. So, diffraction, increasing with $(n-1)$, acts on a very long time, increasing with $m$, before the nonlinear regime could become relevant, and if $(n-1)$ or $m$ is sufficiently large, one expects that the solution would be diffracted away almost completely, before reaching the nonlinear regime, and will not break. We find, in particular, that breaking takes place in the following longtime regimes (see (4.3)):

$$
\begin{aligned}
& m=n=1 \text { (the Riemann equation with quadratic nonlinearity): } t_{\mathrm{b}}=O\left(\epsilon^{-1}\right) \\
& m=1, \quad n=2 \text { (the dKP equation): } t_{\mathrm{b}}=O\left(\epsilon^{-2}\right) ; \\
& m=1, \quad n=3 \text { (the KZ equation): } t_{\mathrm{b}}=O\left(\mathrm{e}^{\epsilon^{-1}}\right) \\
& m=2, \quad n=1 \text { (the Riemann equation with cubic nonlinearity): } t_{\mathrm{b}}=O\left(\epsilon^{-2}\right) \\
& m=2, \quad n=2 \text { (the mdKP equation): } O\left(t_{\mathrm{b}}=\mathrm{e}^{\epsilon^{-2}}\right) .
\end{aligned}
$$

It is interesting that, as observed in [2], if $m=1$ (the physically more relevant case), wave breaking takes place only for $n \leqslant 3$; i.e., in physical space!

We end this section remarking that also in the longtime behavior of the solutions of the small data Cauchy problem for equation (1.1), the dependence of the solution $u$ on $x$, as in the 
case of the exact solutions (3.14), is not consistent with the formal ansatz recently made in [29].

\section{Analytic aspects of wave breaking}

In this section we use the longtime behavior of solutions of the small data Cauchy problem for $\mathrm{dKP}(m, n)$ to show explicitly the analytic aspects of the solution in the neighborhood of the breaking time in terms of the initial data, represented by function $G$ defined in (4.7), as already done in $[2,26]$.

Choosing $c_{m, n} \geqslant 0$, we rewrite equation (4.9) in the characteristic form

$$
\begin{aligned}
& q \sim \epsilon G(\zeta, \vec{\eta}), \\
& \quad \xi=\epsilon^{m} F(\zeta, \vec{\eta} ; m) \tau+\zeta, \quad F(\zeta, \vec{\eta} ; m) \equiv G^{m}(\zeta, \vec{\eta}),
\end{aligned}
$$

where

$$
q=u t^{\frac{n-1}{2}}, \quad \xi=x+\frac{1}{4 t} \sum_{j=1}^{n-1} y_{j}^{2}, \quad \vec{\eta}=\frac{\vec{y}}{2 t}
$$

and

$$
\tau(t)= \begin{cases}\frac{1}{c_{m, n}} t^{c_{m, n},}, & \text { if } c_{m, n}>0 \\ \ln t, & \text { if } c_{m, n}=0\end{cases}
$$

One solves the second equation of (5.1) with respect to the parameter $\zeta$, obtaining $\zeta(\xi, \vec{\eta}, \tau)$, and substitutes it into the first, to obtain the solution $q \sim \epsilon G(\zeta(\xi, \vec{\eta}, \tau), \vec{\eta})$. The inversion of the second equation of (5.1) is possible iff its $\zeta$-derivative is different from zero. Therefore the singularity manifold (SM) of the solution is the $n$-dimensional manifold characterized by the equation

$\mathcal{S}(\zeta, \vec{\eta}, \tau) \equiv 1+\epsilon^{m} F_{\zeta}(\zeta, \vec{\eta}, m) \tau=0 \quad \Rightarrow \quad \tau=-\frac{1}{\epsilon^{m} F_{\zeta}(\zeta, \vec{\eta}, m)}$.

Since

$$
\nabla_{(\xi, \vec{\eta})} q=\frac{\epsilon \nabla_{(\zeta, \vec{\eta}} G(\zeta, \vec{\eta})}{1+\epsilon^{m} F_{\zeta}(\zeta, \vec{\eta}, m) \tau}
$$

the slope of the localized wave becomes infinity (the so-called gradient catastrophe) on the $\mathrm{SM}$, and the $n$-dimensional wave 'breaks' except on the direction represented by the vector field $\hat{V}=\sum_{i=1}^{n-1} \partial_{\eta_{i}}+m \epsilon^{m} G^{m-1}\left(\sum_{i=1}^{n-1} G_{\eta_{i}}\right) \partial_{\xi}[2,27]$, on which:

$$
\hat{V} q=\epsilon \sum_{i=1}^{n-1} G_{\eta_{i}}
$$

Since the $\mathrm{KZ}$ case $(m, n)=(1,3)$ has been studied in detail in [2], in the following we mainly concentrate on the mdKP case $(m, n)=(2,2)$, and we compare it with the dKP case $(m, n)=(1,2)$ investigated in [26].

From (5.4), the first breaking time $\tau_{\mathrm{b}}$ and the corresponding characteristic parameters $\vec{\zeta}_{\mathrm{b}}=\left(\zeta_{\mathrm{b}}, \eta_{\mathrm{b}}\right)$ are defined by the global minimum of a function of the two variables $(\zeta, \eta)$ : 


$$
\tau_{\mathrm{b}}=-\frac{1}{\epsilon^{m} F_{\zeta}\left(\zeta_{\mathrm{b}}, \eta_{\mathrm{b}} ; m\right)}:=\inf \left(-\frac{1}{\epsilon^{m} F_{\zeta}(\zeta, \eta ; m)}\right)>0,
$$

and it is characterized, together with the condition $F_{\zeta}\left(\vec{\zeta}_{\mathrm{b}} ; m\right)<0$, by the following equations:

$$
\begin{aligned}
& F_{\zeta \zeta}\left(\vec{\zeta}_{\mathrm{b}} ; m\right)=F_{\zeta \eta}\left(\vec{\zeta}_{\mathrm{b}} ; m\right)=0 \\
& \quad \alpha \equiv F_{\zeta \zeta \zeta}\left(\vec{\zeta}_{\mathrm{b}} ; m\right) F_{\zeta \eta \eta}\left(\vec{\zeta}_{\mathrm{b}} ; m\right)-F_{\zeta \zeta \eta}^{2}\left(\vec{\zeta}_{\mathrm{b}} ; m\right)>0, \quad F_{\zeta \zeta \zeta}\left(\vec{\zeta}_{\mathrm{b}} ; m\right)>0 .
\end{aligned}
$$

The corresponding point at which the first wave breaking takes place is, from (5.1), $\vec{\xi}_{\mathrm{b}}=\left(\xi_{\mathrm{b}}, \eta_{\mathrm{b}}\right) \in \mathbb{R}^{2}$, where:

$$
\xi_{\mathrm{b}}=\zeta_{\mathrm{b}}+\epsilon^{m} F\left(\vec{\zeta}_{\mathrm{b}} ; m\right) \tau_{\mathrm{b}}=\zeta_{\mathrm{b}}-\frac{F\left(\vec{\zeta}_{\mathrm{b}} ; m\right)}{F_{\zeta}\left(\vec{\zeta}_{\mathrm{b}} ; m\right)} .
$$

Now we evaluate equations (5.1) and (5.4) near breaking, in the regime:

$$
\xi=\xi_{\mathrm{b}}+\xi^{\prime}, \quad \eta=\eta_{\mathrm{b}}+\eta^{\prime}, \quad \tau=\tau_{\mathrm{b}}+\tau^{\prime}, \quad \zeta=\zeta_{\mathrm{b}}+\zeta^{\prime},
$$

where $\xi^{\prime}, \eta^{\prime}, \tau^{\prime}, \zeta^{\prime}$ are small. Using (5.7)-(5.9), the second equation of (5.1) becomes, at the leading order, the following cubic equation in $\zeta^{\prime}$ :

$$
\zeta^{\prime 3}+a\left(\eta^{\prime}\right) \zeta^{\prime 2}+b\left(\eta^{\prime}, \tilde{\tau}\right) \zeta^{\prime}-\gamma X\left(\xi^{\prime}, \eta^{\prime}, \tilde{\tau}\right)=0
$$

where

$$
\begin{aligned}
& a\left(\eta^{\prime}\right)=\frac{3 F_{\zeta \zeta}}{F_{\zeta \zeta \zeta}} \eta^{\prime}, \\
& b\left(\eta^{\prime}, \tilde{\tau}\right)=\frac{3}{F_{\zeta \zeta \zeta}}\left[2 F_{\zeta} \tilde{\tau}+F_{\zeta \eta \eta} \eta^{\prime 2}\right], \\
& X\left(\xi^{\prime}, \eta^{\prime}, \tilde{\tau}\right)=\xi^{\prime}-\epsilon F\left(\zeta_{\mathrm{b}}, \eta_{\mathrm{b}}+\eta^{\prime}\right) \tau^{\prime}-\epsilon\left[F\left(\zeta_{\mathrm{b}}, \eta_{\mathrm{b}}+\eta^{\prime}\right)-F\right] \tau_{\mathrm{b}} \sim \\
& \xi^{\prime}-\frac{F_{\eta}}{\left|F_{\zeta}\right|} \eta^{\prime}-\frac{F}{\left|F_{\zeta}\right|} \tilde{\tau}-\frac{F_{\eta \eta}}{2\left|F_{\zeta}\right|} \eta^{\prime 2}-\frac{F_{\eta}}{\left|F_{\zeta}\right|} \eta^{\prime} \tilde{\tau}-\frac{F_{\eta \eta}}{6\left|F_{\zeta}\right|} \eta^{\prime 3}, \quad \gamma=\frac{6\left|F_{\zeta}\right|}{F_{\zeta \zeta \zeta}},
\end{aligned}
$$

and

$$
\tilde{\tau} \equiv \frac{\tau^{\prime}}{\tau_{\mathrm{b}}}=\frac{\tau-\tau_{\mathrm{b}}}{\tau_{\mathrm{b}}}
$$

corresponding to the maximal balance

$$
\left|\zeta^{\prime}\right|,\left|\eta^{\prime}\right|=O\left(|\tilde{\tau}|^{1 / 2}\right), \quad|X|=O\left(|\tilde{\tau}|^{3 / 2}\right) .
$$

In (5.12) and in the rest of this section, $G, F=G^{m}$ and all their partial derivatives whose arguments are not indicated are meant to be evaluated at $\vec{\zeta}_{\mathrm{b}}=\left(\zeta_{\mathrm{b}}, \eta_{\mathrm{b}}\right)$.

The three roots of the cubic are given by the well-known Cardano-Tartaglia formula:

$$
\begin{aligned}
& \zeta_{0}^{\prime}\left(\xi^{\prime}, \eta^{\prime}, \tilde{\tau}\right)=-\frac{a}{3}+\left(A_{+}\right)^{\frac{1}{3}}+\left(A_{-}\right)^{\frac{1}{3}}, \\
& \zeta_{ \pm}^{\prime}\left(\xi^{\prime}, \eta^{\prime}, \tilde{\tau}\right)=-\frac{a}{3}-\frac{1}{2}\left(\left(A_{+}\right)^{\frac{1}{3}}+\left(A_{-}\right)^{\frac{1}{3}}\right) \pm \frac{\sqrt{3}}{2} \mathrm{i}\left(\left(A_{+}\right)^{\frac{1}{3}}-\left(A_{-}\right)^{\frac{1}{3}}\right),
\end{aligned}
$$

where

$$
A_{ \pm}=R \pm \sqrt{\Delta}
$$

and the discriminant $\Delta$ reads

$$
\Delta=R^{2}+Q^{3}
$$


with

$$
\begin{aligned}
& Q\left(\eta^{\prime}, \tilde{\tau}\right)=\frac{3 b-a^{2}}{9}=-\frac{2\left|F_{\zeta}\right|}{F_{\zeta \zeta \zeta}} \tilde{\tau}+\frac{\alpha}{F_{\zeta \zeta \zeta}^{2}} \eta^{\prime 2} \\
& R\left(\xi^{\prime}, \eta^{\prime}, \tilde{\tau}\right)=\frac{\gamma}{2} X\left(\xi^{\prime}, \eta^{\prime}, \tilde{\tau}\right)+\frac{a b}{18}+\frac{a}{3} Q\left(\eta^{\prime}, \tilde{\tau}\right)
\end{aligned}
$$

At the same order, function $\mathcal{S}$ in (5.4) becomes

$$
\begin{aligned}
& \mathcal{S}\left(\zeta^{\prime}, \eta^{\prime}, \tilde{\tau}\right)=-\tilde{\tau}+\frac{1}{2\left|F_{\zeta}\right|}\left[F_{\zeta \zeta \zeta} \zeta^{\prime 2}+2 F_{\zeta \zeta \eta} \zeta^{\prime} \eta^{\prime}+F_{\zeta \eta \eta} \eta^{\prime 2}\right] \\
& =\frac{F_{\zeta \zeta \zeta}}{2\left|F_{\zeta}\right|}\left(Q\left(\eta^{\prime}, \tilde{\tau}\right)+\left(\zeta^{\prime}+\frac{F_{\zeta \zeta \eta}}{F_{\zeta \zeta \zeta}} \eta^{\prime}\right)\right.
\end{aligned}
$$

Known $\zeta^{\prime}$ as the function of $\left(\xi^{\prime}, \eta^{\prime}, \tilde{\tau}\right)$ from the cubic (5.11), the solution $q$ and its gradient are then approximated, near breaking, by the formulae:

$$
\begin{aligned}
q(\xi, \eta, \tau) & \sim \epsilon G\left(\zeta_{\mathrm{b}}+\zeta^{\prime}, \eta_{\mathrm{b}}+\eta^{\prime}\right)=\epsilon\left(G+G_{\zeta} \zeta^{\prime}+G_{\eta} \eta^{\prime}+O(|\tilde{\tau}|)\right), \\
\nabla_{(\xi, \eta)} q & \sim \epsilon \frac{\nabla_{\left(\zeta^{\prime}, \eta^{\prime}\right)} G\left(\zeta_{\mathrm{b}}+\zeta^{\prime}, \eta_{\mathrm{b}}+\eta^{\prime}\right)}{\mathcal{S}\left(\zeta^{\prime}, \eta^{\prime}, \tilde{\tau}\right)} .
\end{aligned}
$$

\section{Before breaking}

Before breaking: $\tau<\tau_{\mathrm{b}}(\tilde{\tau}<0)$, the coefficient $Q$ in $(5.18)$ is strictly positive (see the second equation of (5.8)); then the discriminant $\Delta=R^{2}+Q^{3}$ is also strictly positive and only the $\operatorname{root} \zeta_{0}^{\prime}$ is real. Correspondingly, the real solution $q$ is single-valued and described by Cardano's formula and by (5.20). In addition, function $\mathcal{S}$ in (5.19) is also strictly positive and $\nabla_{(\xi, \vec{\eta})} q$ is finite $\forall \xi, \eta$.

To have a more explicit solution than that provided by Cardano's formula, we first restrict the asymptotic region to a narrower area, so that the cubic (5.11) reduces to the linear equation $b \zeta^{\prime}=\gamma X$, and $X$ contains only linear terms. This is achieved by choosing:

$$
\eta^{\prime}=O\left(\tilde{\tau}^{r}\right), X=O\left(\tilde{\tau}^{p+1}\right), \frac{1}{2}<p<\frac{p+1}{2}<r<p+1
$$

implying

$$
\begin{aligned}
\zeta^{\prime} & =O\left(\tilde{\tau}^{p}\right), \\
X & =\xi^{\prime}+\frac{F_{\eta}}{F_{\zeta}} \eta^{\prime}+\frac{F}{F_{\zeta}} \tilde{\tau}+o\left(\tilde{\tau}^{p+1}\right)=\xi^{\prime}+\frac{G_{\eta}}{G_{\zeta}} \eta^{\prime}+\frac{G}{m G_{\zeta}} \tilde{\tau}+o\left(\tilde{\tau}^{p+1}\right), \\
b \zeta^{\prime} & =\gamma X \Rightarrow \zeta^{\prime} \sim-\frac{1}{\tilde{\tau}}\left(\xi^{\prime}+\frac{G_{\eta}}{G_{\zeta}} \eta^{\prime}+\frac{G}{m G_{\zeta}} \tilde{\tau}\right)
\end{aligned}
$$

Then

$q=\epsilon G\left(\zeta_{\mathrm{b}}+\zeta^{\prime}, \eta_{\mathrm{b}}+\eta^{\prime}\right) \sim \epsilon G+\epsilon G_{\zeta} \zeta^{\prime} \sim \epsilon\left(1-\frac{1}{m}\right) G-\frac{\epsilon G_{\zeta}}{\tilde{\tau}}\left(\xi^{\prime}+\frac{G_{\eta}}{G_{\zeta}} \eta^{\prime}\right)$.

Since $q \sim \epsilon G$, and recalling the definitions (5.7), (5.13), it follows that $q$ is described by the exact similarity solution

$$
q \sim\left(\frac{\xi+\left(G_{\eta} / G_{\zeta}\right) \eta^{\prime}}{\tau^{\prime}}\right)^{\frac{1}{m}}
$$

of equation (3.7). 
The cubic is also simplified in the asymptotic region in which $\xi^{\prime}, \eta^{\prime}$ are of the same order while $\tau^{\prime} \leqslant O\left(\left|\eta^{\prime}\right|\right)$, reducing to $\zeta^{\prime 3}=\gamma X$, so that the solution $q$ and its gradient are

$$
\begin{aligned}
& q \sim \epsilon G\left(\zeta_{\mathrm{b}}+\sqrt[3]{\gamma X\left(\xi^{\prime}, \eta^{\prime}, \tilde{\tau}\right)}, \eta_{\mathrm{b}}+\eta^{\prime}\right) \\
& \nabla_{\xi, \eta} q \sim \frac{\sqrt[3]{\gamma}}{3} \frac{\epsilon \nabla_{\zeta, \eta} G}{\sqrt[3]{X^{2}\left(\xi^{\prime}, \eta^{\prime}, \tilde{\tau}\right)}} .
\end{aligned}
$$

\section{At breaking}

When $\tau=\tau_{\mathrm{b}}$, the $\operatorname{SM} \mathcal{S}$ (5.19) is positive everywhere in the $(\xi, \eta)$ plane, except at the breaking point $\left(\xi_{\mathrm{b}}, \eta_{\mathrm{b}}\right)$, in which it is zero, together with $Q, R$ and the discriminant $\Delta$. The solution $q$ is well described again by Cardano's formula and by (5.20). To have a more explicit solution than that provided by Cardano's formula, we choose $\xi^{\prime}, \eta^{\prime}$ of the same order, the cubic (5.11) reduces to $\zeta^{\prime 3}=\gamma X$ and equations (5.25) become

$$
\begin{aligned}
& q \sim \epsilon G\left(\zeta_{\mathrm{b}}+\sqrt[3]{\gamma X\left(\xi^{\prime}, \eta^{\prime}, 0\right)}, \eta_{\mathrm{b}}+\eta^{\prime}\right) \\
& \nabla_{\xi, \eta} q \sim \frac{\sqrt[3]{\gamma}}{3} \frac{\epsilon \nabla_{\xi, \eta} G}{\sqrt[3]{X^{2}\left(\xi^{\prime}, \eta^{\prime}, 0\right)}}
\end{aligned}
$$

The expression of $X_{\mathrm{b}}\left(\xi^{\prime}, \vec{\eta}^{\prime}\right) \equiv X\left(\xi^{\prime}, \vec{\eta}^{\prime}, 0\right)$ is given in (5.12): $X_{\mathrm{b}}\left(\xi^{\prime}, \eta^{\prime}\right) \sim \xi^{\prime}-\frac{F_{\eta}}{\left|F_{\zeta}\right|} \eta^{\prime}$ if $\xi^{\prime} \propto \eta^{\prime}$ but $\xi^{\prime} \neq\left(F_{\eta} / F_{\zeta}\right) \eta^{\prime}$, while $X\left(\xi^{\prime}, \eta^{\prime}, 0\right)=-\frac{F_{\eta \eta}}{2\left|F_{\zeta}\right|} \eta^{\prime 2}$ if $\xi^{\prime}=\left(F_{\eta} / F_{\zeta}\right) \eta^{\prime}$.

Equation (5.27) implies that all the derivatives of $q$ blow up at $\tau=\tau_{\mathrm{b}}$, in the breaking point $\left(\xi_{\mathrm{b}}, \eta_{\mathrm{b}}\right)$, with the universal law $X_{\mathrm{b}}^{-2 / 3}$, except the derivative along the transversal line $X_{\mathrm{b}}\left(\xi^{\prime}, \eta^{\prime}, 0\right)=0$, represented by the vector field $\hat{V}=\partial_{\eta}-X_{\mathrm{b} \eta} \partial_{\xi}$ (compare with (5.6)), for which $[2,27]$

$$
\left.\hat{V} q\right|_{\left(\xi_{\mathrm{b}}, \eta_{\mathrm{b}}\right)}=\epsilon G_{\eta}
$$

\section{After breaking l: the overturning profile}

After breaking, the solution becomes three-valued in a compact region of the $(\xi, \eta)$ plane (see figures 6,7), and, in general, does not describe any physics; nevertheless a detailed study of the multivalued region is important, in view of a proper regularization of the models.

If $\tau>\tau_{\mathrm{b}}(\tilde{\tau}>0)$, in the regime (5.14), the $\mathrm{SM}$ equation $\mathcal{S}=0$ :

$$
2\left|F_{\zeta}\right| \tilde{\tau}=F_{\zeta \zeta \zeta} \zeta^{\prime 2}+2 F_{\zeta \zeta \eta} \zeta^{\prime} \eta^{\prime}+F_{\zeta \eta \eta} \eta^{\prime 2}
$$

describes an ellipsoidal paraboloid in the $\left(\zeta^{\prime}, \eta^{\prime}, \tilde{\tau}\right)$ space, with the minimum at the breaking point $\left(\vec{\xi}_{\mathrm{b}}, \tilde{\tau}_{\mathrm{b}}\right)$.

One can check that, eliminating $\zeta^{\prime}$ from equations (5.29) and (5.11), one obtains the SM equation in space-time coordinates, coinciding with the equation $\Delta=R^{2}+Q^{3}=0$, where $\Delta$ is the discriminant (5.17) of the cubic (5.11), and $Q$ and $R$ are defined in (5.18). Such an equation can be satisfied only if $Q \leqslant 0$; i.e., only if

$$
\left|\eta^{\prime}\right| \leqslant \eta_{\text {cusp }}^{\prime}(\tilde{\tau})
$$



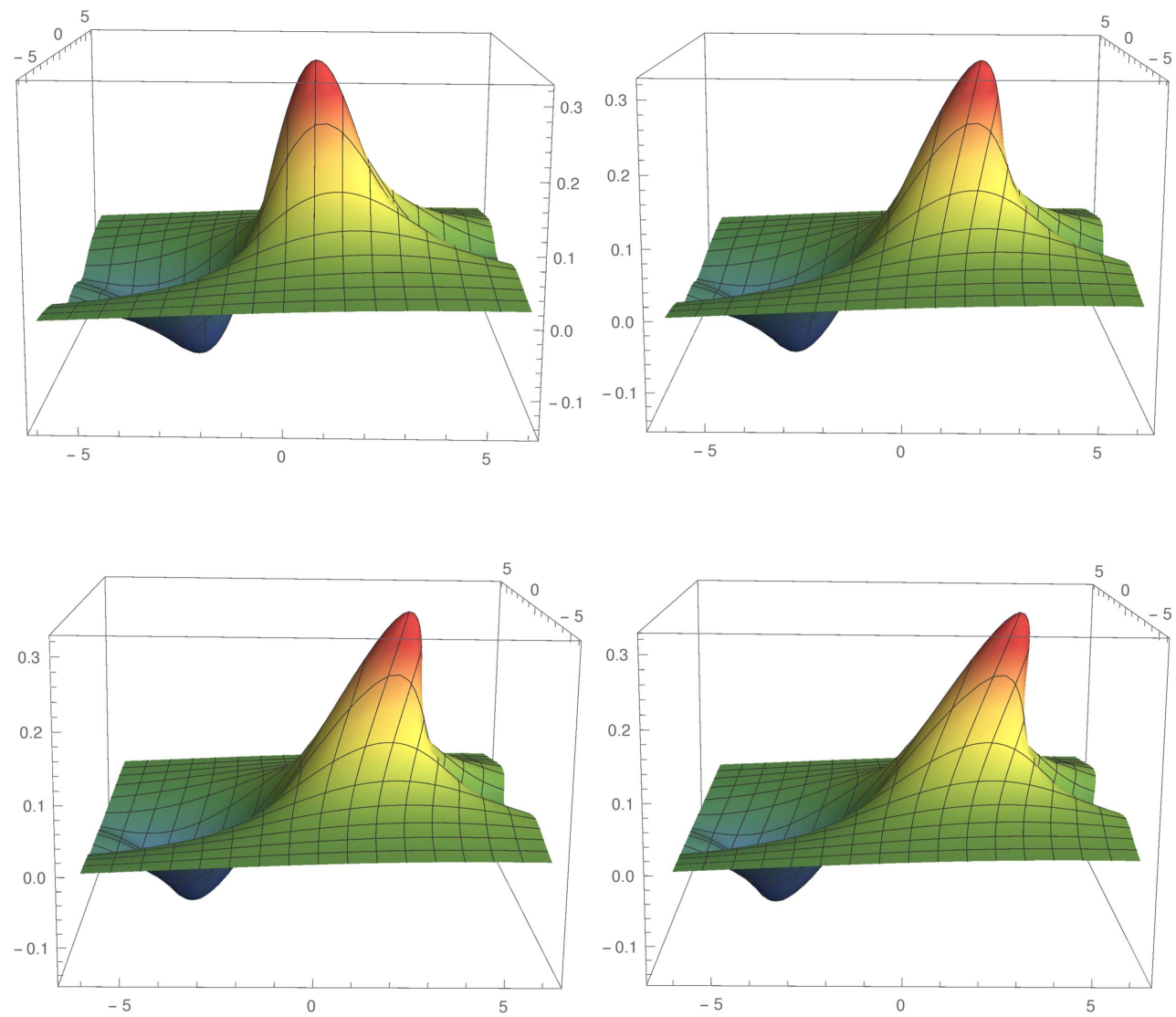

Figure 4. Plotting of the analytic solution $q(\xi, \eta, \tau)$ defined in (5.20) corresponding to $\mathrm{dKP}(m=1)$, for $G$ given by (4.22), $\epsilon=0.2$, at times $\tau=0, \quad \tau_{\mathrm{b}}-2, \quad \tau_{\mathrm{b}}, \quad \tau_{\mathrm{b}}+2$., where $\tau_{\mathrm{b}}=6.57$.

where

$$
\eta_{\text {cusp }}^{\prime}(\tilde{\tau}) \equiv \sqrt{\frac{2\left|F_{\zeta}\right| F_{\zeta \zeta \zeta}}{\alpha}} \sqrt{\tilde{\tau}}
$$

and is given by the following analytic and universal expression

$$
\begin{aligned}
& \left\{\xi^{\prime}-\frac{F_{\eta}}{\left|F_{\zeta}\right|} \eta^{\prime}-\frac{F}{\left|F_{\zeta}\right|} \tilde{\tau}-\frac{F_{\eta \eta}}{2\left|F_{\zeta}\right|} \eta^{\prime 2}-\left(\frac{F_{\eta}}{\left|F_{\zeta}\right|}+\frac{F_{\zeta \zeta \eta}}{F_{\zeta \zeta \zeta}}\right) \eta^{\prime} \tilde{\tau}+\frac{1}{6\left|F_{\zeta}\right| F_{\zeta \zeta \zeta}^{2}}\left[-F_{\eta \eta \eta} F_{\zeta \zeta \zeta}^{2}\right.\right. \\
& \left.\left.+F_{\zeta \zeta \eta}\left(3 F_{\zeta \zeta \zeta} F_{\zeta \eta \eta}-2 F_{\zeta \zeta \eta}^{2}\right)\right] \eta^{\prime 3}\right\}^{2}=\frac{\alpha^{3}}{9 F_{\zeta}^{2} F_{\zeta \zeta \zeta}^{4}}\left(\left(\eta_{\text {cusp }}^{\prime}(\tilde{\tau})\right)^{2}-\eta^{\prime 2}\right)^{3} .
\end{aligned}
$$

Equation (5.32) describes a closed curve with two cusps in the $(\xi, \eta)$ plane at the points

$$
\vec{\xi}_{\text {cusp }}^{ \pm}(\tilde{\tau})=\left(\xi_{\mathrm{b}}, \eta_{\mathrm{b}}\right)+\left(\xi_{\text {cusp }}^{ \pm^{\prime}}(\tilde{\tau}), \pm \eta_{\text {cusp }}^{\prime}(\tilde{\tau})\right)
$$



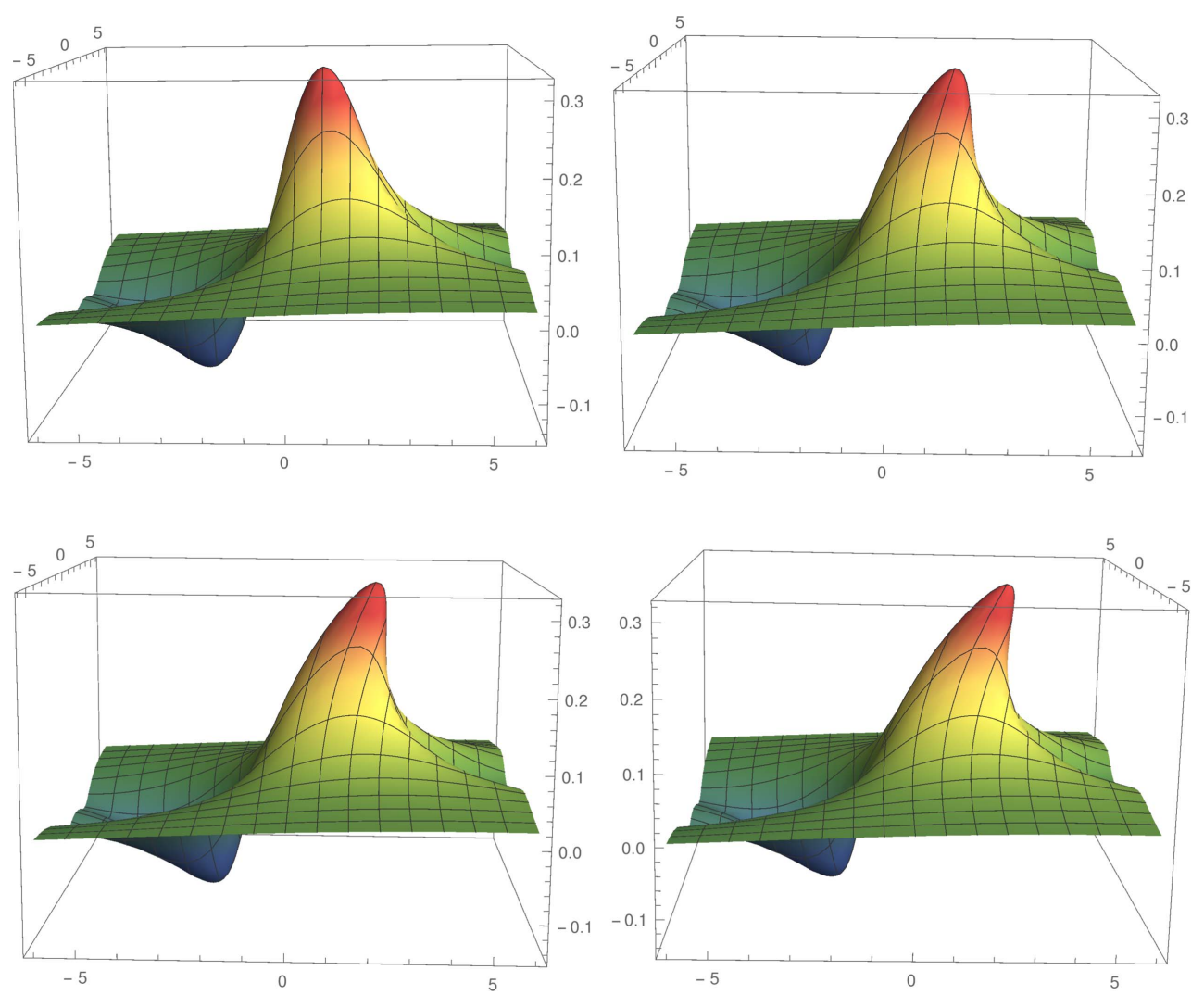

Figure 5. Plotting of the analytic solution $q(\xi, \eta, \tau)$ defined in (5.20) corresponding to $\operatorname{mdKP}(m=2)$, for $G$ given by (4.22), $\epsilon=0.2$, at times $\tau=0, \quad \tau_{\mathrm{b}}-4, \quad \tau_{\mathrm{b}}, \quad \tau_{\mathrm{b}}+4$, where $\tau_{\mathrm{b}}=14.31$.

where

$$
\xi_{\text {cusp }}^{ \pm^{\prime}}(\tilde{\tau}) \sim \pm F_{\eta} \sqrt{\frac{2 F_{\zeta \zeta \zeta}}{\alpha\left|F_{\zeta}\right|}} \sqrt{\tilde{\tau}}+\left(\frac{F}{\left|F_{\zeta}\right|}+\frac{F_{\eta \eta} F_{\zeta \zeta \zeta}}{\alpha}\right) \tilde{\tau} \pm B \tilde{\tau}^{3 / 2}
$$

where $B=O(1)$ (see figure 6 for $\mathrm{dKP}$ and 7 for mdKP), corresponding to the conditions $Q=R=0$, on which the three real solutions of the cubic coincide (in $1+1$ dimensions, it is not possible to have three coincident solutions!):

$$
\zeta_{0}=\zeta_{ \pm}=-\frac{F_{\zeta \zeta \eta}}{F_{\zeta \zeta \zeta}} \eta^{\prime}, \Rightarrow q_{0}=q_{ \pm}=\sim \epsilon\left(G+\left(G_{\eta}-G_{\zeta} \frac{F_{\zeta \zeta \eta}}{F_{\zeta \zeta \zeta}}\right) \eta^{\prime}\right) .
$$

On the remaining part of the closed curve $\Delta=0$, two of the three real branches coincide. Outside the closed curve, $\Delta>0$ and the real solution $q$ is single valued; inside the closed curve, $\Delta<0$ and the real solution $q$ is three valued, with the three branches

$$
q=\epsilon G\left(\zeta_{\mathrm{b}}+\zeta^{\prime}, \eta_{\mathrm{b}}+\eta^{\prime}\right) \sim \epsilon\left(G+G_{\zeta} \zeta^{\prime}+G_{\eta} \eta^{\prime}+O(\tilde{\tau})\right),
$$

where $\zeta^{\prime}$ represents the three real solutions of the cubic (5.11) (see figure 4 for $\mathrm{dKP}$ and figure 5 for $\mathrm{mdKP}$ ). 

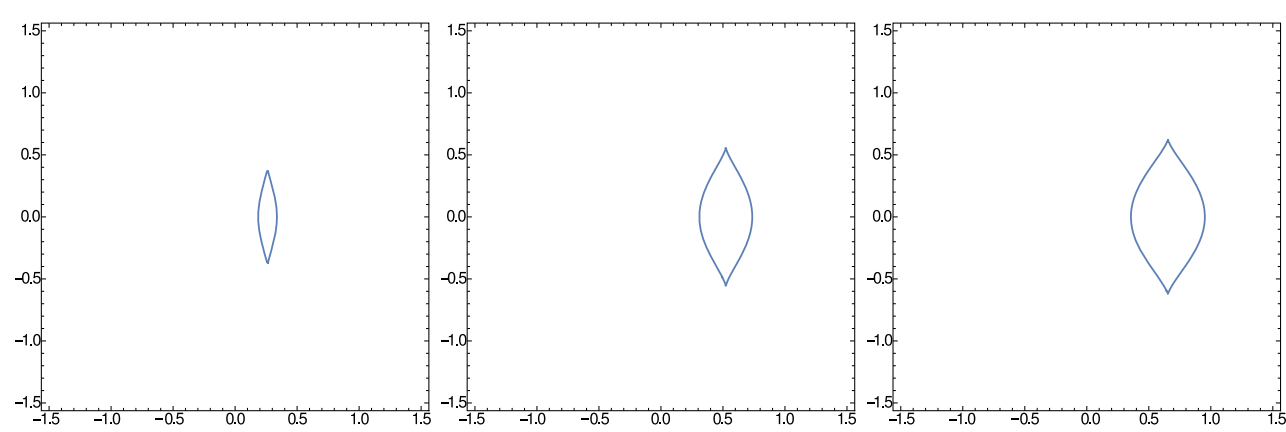

Figure 6. Plotting of the three-valued region of the $(\xi, \eta)$ plane for dKP at times $\tilde{\tau}=0.2, \quad 0.4, \quad 0.5$ for $G$ given by (4.22) and $\epsilon=0.2$. The center of the square frame is the breaking point $\left(\xi_{\mathrm{b}}, \eta_{\mathrm{b}}\right)$. Notice that the two cusps have the same $\xi$ coordinate $\xi_{\text {cusp }}^{+}(\tilde{\tau}) \sim \xi_{\text {cusp }}^{-}(\tilde{\tau})$ since, for solution (4.22), $G_{\eta}=0$ (see (5.31), (5.34)).
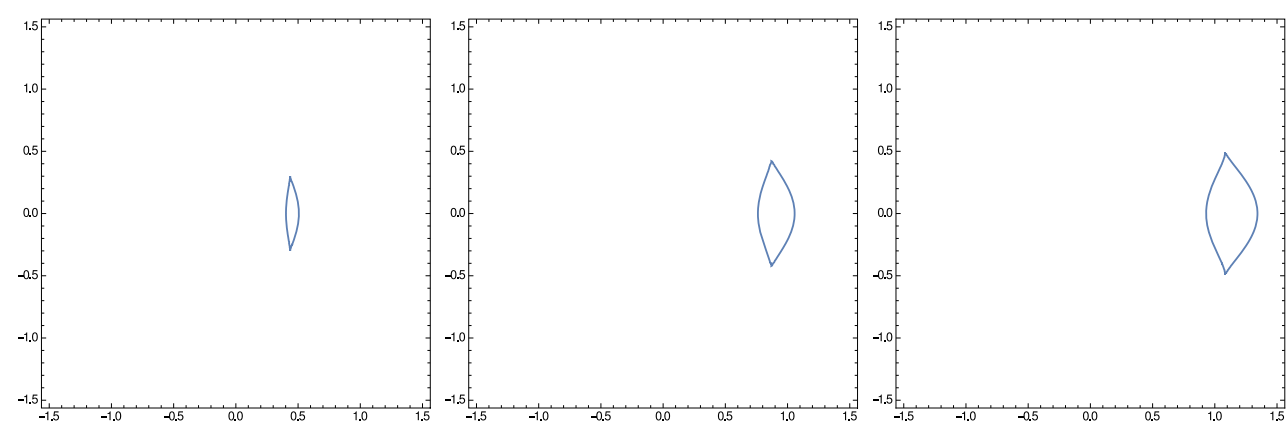

Figure 7. Plotting of the three-valued region of the $(\xi, \eta)$ plane for mdKP at times $\tilde{\tau}=0.2, \quad 0.4, \quad 0.5$ for $G$ given by (4.22) and $\epsilon=0.2$. The center of the square frame is the breaking point $\left(\xi_{\mathrm{b}}, \eta_{\mathrm{b}}\right)$. Notice that the two cusps have the same $\xi$ coordinate $\xi_{\text {cusp }}^{+}(\tilde{\tau}) \sim \xi_{\text {cusp }}^{-}(\tilde{\tau})$ since, for solution (4.22), $F_{\eta}=G_{\eta}=0$ (see (5.31), (5.34)).

Such a three-valued region of the $(\xi, \eta)$ plane is characterized by the conditions

$$
\left|\eta^{\prime}\right|<\eta_{\text {cusp }}^{\prime}(\tilde{\tau}), \quad \xi_{M}^{-}\left(\eta^{\prime}, \tilde{\tau}\right)<\xi^{\prime}<\xi_{M}^{+}\left(\eta^{\prime}, \tilde{\tau}\right)
$$

where

$$
\begin{aligned}
& \xi_{M}^{ \pm}\left(\eta^{\prime}, \tilde{\tau}\right) \equiv \frac{F_{\eta}}{\left|F_{\zeta}\right|} \eta^{\prime}+\frac{F}{\left|F_{\zeta}\right|} \tilde{\tau}+\frac{F_{\eta \eta}}{2\left|F_{\zeta}\right|} \eta^{\prime 2}+\left(\frac{F_{\eta}}{\left|F_{\zeta}\right|}+\frac{F_{\zeta \zeta \eta}}{F_{\zeta \zeta \zeta}}\right) \eta^{\prime} \tilde{\tau}-\frac{1}{6\left|F_{\zeta}\right| F_{\zeta \zeta \zeta}^{2}}\left[-F_{\eta \eta \eta} F_{\zeta \zeta \zeta}^{2}\right. \\
& \left.\quad+F_{\zeta \zeta \eta}\left(3 F_{\zeta \zeta \zeta} F_{\zeta \eta \eta}-2 F_{\zeta \zeta \eta}^{2}\right)\right] \eta^{\prime 3} \pm \frac{F_{\zeta \zeta \zeta}}{3\left|F_{\zeta}\right|}|Q|^{\frac{3}{2}}
\end{aligned}
$$

(see figure 6 for dKP and figure 7 for mdKP).

Since

$\Delta \xi \equiv \xi_{M}^{+}\left(\eta^{\prime}, \tilde{\tau}\right)-\xi_{M}^{-}\left(\eta^{\prime}, \tilde{\tau}\right)=\frac{2 F_{\zeta \zeta \zeta}}{3\left|F_{\zeta}\right|}\left|Q\left(\eta^{\prime}, \tilde{\tau}\right)\right|^{3 / 2} \leqslant \frac{4 \sqrt{2}}{3} \sqrt{\frac{\left|F_{\zeta}\right|}{F_{\zeta \zeta \zeta}}} \tilde{\tau}^{3 / 2}$,

we infer that the longitudinal width of the multivalued region is $O\left(\tilde{\tau}^{3 / 2}\right)$; the transversal width delimited by the two cusps is instead $O\left(\tilde{\tau}^{1 / 2}\right)$; therefore the multivalued region develops, at 
$\tau=\tau_{\mathrm{b}}$, from the breaking point $\left(\xi_{\mathrm{b}}, \eta_{\mathrm{b}}\right)$, with an infinite speed in the transversal direction, and with zero speed in the longitudinal direction. All these results are a common feature to the class of dynamics (1.1) (see the previously derived results for the dKP equation, for finite time $[26,27]$ and in the longtime regime [26], and those for the $\mathrm{KZ}$ equation in the longtime regime [2]).

We end this subsection remarking that, as already observed in [27], since $\operatorname{dKP}(m, n)$ is symmetric w.r.t. $y$, if the initial datum is also symmetric w.r.t. $y$, like the Gaussian (4.19), its evolution will also be so: $u(x, y, t)=u(x,-y, t)$. This implies that $\eta_{\mathrm{b}}=y_{\mathrm{b}}=0$, and that all partial derivatives of $F$ and $G$ containing an odd number of $\eta$ derivatives are 0 at breaking. As a consequence, the transversal direction in which the derivative of the profile does not blow up is the $\eta(y)$ direction, and, in it, the derivative is 0 at breaking (see (5.6)).

\section{After breaking II: the shock solution}

As in the $(1+1)$-dimensional case of equation (1.2) [3], after the appearance of the singularity at $t=t_{\mathrm{b}}$, the solution of (1.1) bifurcates into two different solutions: the regular but multivalued solution described above, which may not be acceptable from physical considerations, and a weak discontinuous solution, describing the development of a shock of a dissipative nature.

Using the matching rules of section 3, the shock formulae (3.10) become

$$
\begin{aligned}
& \frac{\partial s}{\partial \tau}=\frac{\epsilon^{m}}{m+1} \frac{G^{m+1}\left(\zeta_{1}, \eta\right)-G^{m+1}\left(\zeta_{2}, \eta\right)}{G\left(\zeta_{1}, \eta\right)-G\left(\zeta_{2}, \eta\right)}, \\
& s=\zeta_{1}+\epsilon^{m} G^{m}\left(\zeta_{1}, \eta\right) \tau=\zeta_{2}+\epsilon^{m} G^{m}\left(\zeta_{2}, \eta\right) \tau
\end{aligned}
$$

where subscript 2 is for values behind the shock and subscript 1 for values ahead. Equations (5.41) are two implicit equations for $\zeta_{1}$ and $\zeta_{2}$ : solving these equations yields $\zeta_{1}(s, \eta, \tau), \zeta_{2}(s, \eta, \tau)$, and, substituting these expressions in (5.40) results in a nonlinear firstorder differential equation for $s$. This equation and the initial conditions (3.11) uniquely give $s(\eta, \tau)$ and, consequently, $\check{\zeta}_{1,2}(\eta, \tau)=\zeta_{1,2}(s(\eta, \tau), \eta, \tau)$.

We recall that, immediately after breaking and if (5.30) holds, equation (5.41) reduces to the cubic (5.11) and (5.12), in which $\xi^{\prime}$ must be replaced by $s^{\prime}$, possessing three real roots, where

$$
s^{\prime}\left(\eta^{\prime}, \tilde{\tau}\right)=s-\xi_{\mathrm{b}} .
$$

Therefore the values of $\zeta_{1}$ and $\zeta_{2}$ are chosen among the three solutions (5.15) so that $q_{2}=\epsilon G\left(\zeta_{\mathrm{b}}+\zeta_{2}^{\prime}, \eta\right)$ has the maximum value and $q_{1}=\epsilon G\left(\zeta_{\mathrm{b}}+\zeta_{1}^{\prime}, \eta\right)$ the minimum one among the three branches of $q$. Since $G_{\zeta}<0$, it follows that

$$
\zeta_{1}=\max \left\{\zeta_{0}^{\prime}, \zeta_{ \pm}^{\prime}\right\} \quad \zeta_{2}=\min \left\{\zeta_{0}^{\prime}, \zeta_{ \pm}^{\prime}\right\}
$$

To identify $\zeta_{1,2}$ with one of the three roots $\zeta_{0}^{\prime}, \zeta_{ \pm}^{\prime}$, let us rewrite them as follows

$$
\begin{aligned}
& \zeta_{0}^{\prime}=-\frac{a}{3}+2 \sqrt[3]{\rho} \cos \left(\frac{\theta}{3}\right), \\
& \zeta_{+}^{\prime}=-\frac{a}{3}-\sqrt[3]{\rho}\left(\cos \left(\frac{\theta}{3}\right)+\sqrt{3} \sin \left(\frac{\theta}{3}\right)\right),
\end{aligned}
$$




$$
\zeta_{-}^{\prime}=-\frac{a}{3}-\sqrt[3]{\rho}\left(\cos \left(\frac{\theta}{3}\right)-\sqrt{3} \sin \left(\frac{\theta}{3}\right)\right)
$$

where

$$
\rho:=\sqrt{R^{2}+|\Delta|}, \quad \theta:=\arctan \left(\frac{\sqrt{|\Delta|}}{R}\right) .
$$

We observe that, for $0 \leqslant \theta \leqslant \pi$, the following inequalities hold

$$
-\cos \left(\frac{\theta}{3}\right)-\sqrt{3} \sin \left(\frac{\theta}{3}\right) \leqslant-\cos \left(\frac{\theta}{3}\right)+\sqrt{3} \sin \left(\frac{\theta}{3}\right) \leqslant 2 \cos \left(\frac{\theta}{3}\right),
$$

so that

$$
\zeta_{+}^{\prime} \leqslant \zeta_{-}^{\prime} \leqslant \zeta_{0}^{\prime} \Rightarrow q\left(\zeta_{\mathrm{b}}+\zeta_{0}^{\prime}, \eta\right) \leqslant q\left(\zeta_{\mathrm{b}}+\zeta_{-}^{\prime}, \eta\right) \leqslant q\left(\zeta_{\mathrm{b}}+\zeta_{+}^{\prime}, \eta\right),
$$

for $q$ decreasing in $\zeta$. Hence

$$
\zeta_{1}^{\prime}\left(s^{\prime}, \eta^{\prime}, \tau^{\prime}\right)=\zeta_{0}^{\prime}\left(s^{\prime}, \eta^{\prime}, \tau^{\prime}\right), \quad \zeta_{2}^{\prime}\left(s^{\prime}, \eta^{\prime}, \tau^{\prime}\right)=\zeta_{+}^{\prime}\left(s^{\prime}, \eta^{\prime}, \tau^{\prime}\right) .
$$

Then (5.40) reduces to the equations

$$
\begin{aligned}
& \frac{\mathrm{d} s^{\prime}}{\mathrm{d} \tilde{\tau}}+\frac{G}{G_{\zeta}}+\frac{G_{\eta}}{G_{\zeta}} \eta^{\prime}+\frac{1}{2}\left[\zeta_{0}^{\prime}\left(s^{\prime}, \eta^{\prime}, \tilde{\tau}\right)+\zeta_{+}^{\prime}\left(s^{\prime}, \eta^{\prime}, \tilde{\tau}\right)\right]=0, \quad \text { for dKP, } \\
& \frac{\mathrm{d} s^{\prime}}{\mathrm{d} \tilde{\tau}}+\frac{G}{2 G_{\zeta}}+\frac{G_{\eta}}{G_{\zeta}} \eta^{\prime}+\frac{1}{2}\left[\zeta_{0}^{\prime}\left(s^{\prime}, \eta^{\prime}, \tilde{\tau}\right)+\zeta_{+}^{\prime}\left(s^{\prime}, \eta^{\prime}, \tilde{\tau}\right)\right]=0, \quad \text { for mdKP, } \\
& s^{\prime}(0)=\xi_{\mathrm{b}},
\end{aligned}
$$

obtained after expanding (5.40) near the breaking point and recalling that $\tilde{\tau} \equiv \tau^{\prime} / \tau_{\mathrm{b}}$.

We remark that, in the differential equations (5.50), $s^{\prime}$ depends on $\eta^{\prime}$ parametrically. So, the dissipative shocks of the longtime solutions of the small data Cauchy problems for dKP and mdKP have a simple geometric construction: given $\tilde{\tau}>0$, for any fixed $\left|\eta^{\prime}\right| \leqslant \eta_{\text {cusp }}$, the overturning profile in the $(\xi, q)$ plane is intercepted by a vertical straight line cutting off equal-area lobi, in analogy with the $1+1$ dimensional case.

\section{Going back to the dKP variables}

The above formulae, written in the convenient variables $q, \xi, \eta, \tau$, are essentially the same for both dKP and mdKP; relevant differences appear when we go back to the original variables $u, x, y, t$ inverting equations (5.2) and (5.3):

$$
\begin{aligned}
& u=\frac{1}{\sqrt{t}} q, \quad t=\frac{\tau^{2}}{4}, \quad y=\frac{\eta \tau^{2}}{2}, \quad x=\xi-\frac{\eta^{2} \tau^{2}}{4}, \quad \text { for dKP, } \\
& u=\frac{1}{\sqrt{t}} q, \quad t=\mathrm{e}^{\tau}, \quad y=2 \eta \mathrm{e}^{\tau}, \quad x=\xi-\eta^{2} \mathrm{e}^{\tau}, \quad \text { for mdKP },
\end{aligned}
$$

and all the above universal formulae are easily transferred to the $\operatorname{dKP}(m, n)$ equations. In particular: small and localized initial data break, (i) at $t_{\mathrm{b}}=\tau_{\mathrm{b}}^{2} / 4$ in the point $\left(x_{\mathrm{b}}, y_{\mathrm{b}}\right)=\left(\xi_{\mathrm{b}}-\tau_{\mathrm{b}}^{2} \eta_{\mathrm{b}}^{2} / 4, \eta_{\mathrm{b}} \tau_{\mathrm{b}}^{2} / 2\right)$ of the parabolic wave front $x+y^{2} /\left(4 t_{\mathrm{b}}\right)=\xi_{\mathrm{b}}$, evolving according to $\mathrm{dKP}$, and (ii) at $t_{\mathrm{b}}=\mathrm{e}^{\tau_{\mathrm{b}}}$ in the point $\left(x_{\mathrm{b}}, y_{\mathrm{b}}\right)=\left(\xi_{\mathrm{b}}-\eta_{\mathrm{b}}^{2} \mathrm{e}^{\tau_{\mathrm{b}}}, 2 \eta_{\mathrm{b}} \mathrm{e}^{\tau_{\mathrm{b}}}\right)$ of the parabolic wave front $x+y^{2} /\left(4 t_{\mathrm{b}}\right)=\xi_{\mathrm{b}}$, evolving according to mdKP. If, for instance, we use the same Gaussian initial condition (4.19) for both dKP and mdKP, with $\epsilon=0.2$, then $G$ is given by (4.22) in both cases. Consequently, for dKP: 

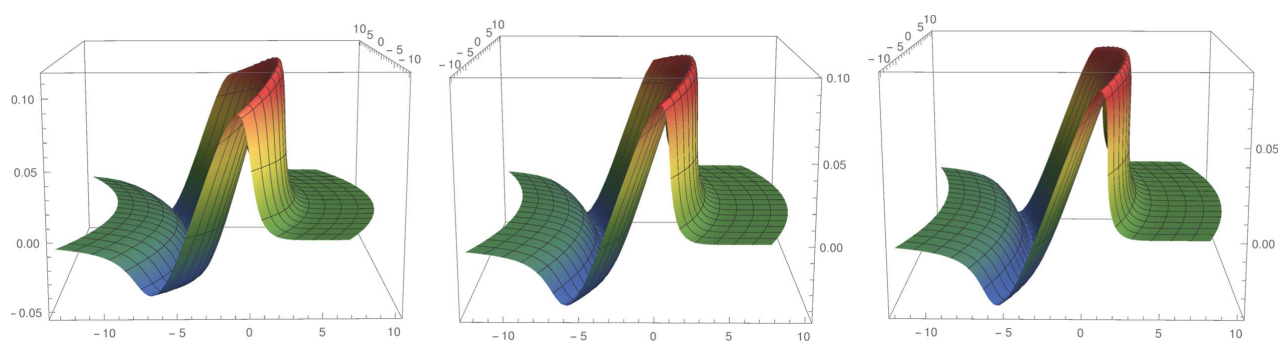

Figure 8. Plotting of the analytic dKP solution $u$ in the physical variables $x, y, t$, for $G$ given by (4.22) (corresponding to the Gaussian initial condition), $\epsilon=0.2$, at times $t=t_{\mathrm{b}}-3, \quad t_{\mathrm{b}}, \quad t_{\mathrm{b}}+3$, where $t_{\mathrm{b}}=10.8$.

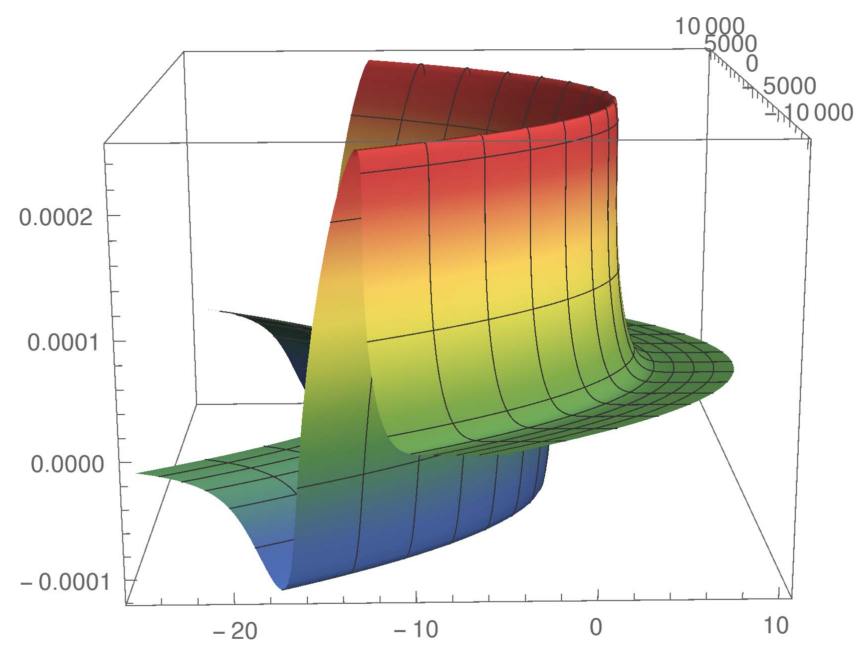

Figure 9. Plotting of the analytic mdKP solution $u$ in the physical variables $x, y, t$, for $G$ given by (4.22) (corresponding to the Gaussian initial condition), $\epsilon=0.2$, at breaking.

$\zeta_{\mathrm{b}}=1.96, \quad \eta_{\mathrm{b}}=0, \quad \xi_{\mathrm{b}}=3.27, \quad \tau_{\mathrm{b}}=6.57$

and wave breaking takes place at $t_{\mathrm{b}}=10.8$ in the point $\left(x_{\mathrm{b}}, y_{\mathrm{b}}\right)=(3.27,0)$ on the parabola $x+2.31 \cdot 10^{-2} y^{2}=3.27$, and at breaking the amplitude of the wave is $u \sim \epsilon t_{\mathrm{b}}{ }^{-1 / 2} G\left(\zeta_{\mathrm{b}}, \eta_{\mathrm{b}}\right)=6.02 \cdot 10^{-2}$ (see figure 8 ).

For mdKP:

$$
\zeta_{\mathrm{b}}=1.61, \quad \eta_{\mathrm{b}}=0, \quad \xi_{\mathrm{b}}=2.51, \quad \tau_{\mathrm{b}}=14.31
$$

and wave breaking takes place at $t_{\mathrm{b}}=1.64 \cdot 10^{6}$ in the point $\left(x_{\mathrm{b}}, y_{\mathrm{b}}\right)=(2.51,0)$ on the parabola $x+1.52 \cdot 10^{-7} y^{2}=2.51$, and at breaking the amplitude of the wave is $u=\epsilon_{\mathrm{b}}{ }^{-1 / 2} G\left(\zeta_{\mathrm{b}}, \eta_{\mathrm{b}}\right)=1.95 \cdot 10^{-4}$ (see figure 9).

Since, in the mdKP case, $t_{\mathrm{b}}$ is very large and the wave amplitude at breaking is very small, we wonder if such wave breaking could be actually detected numerically. 

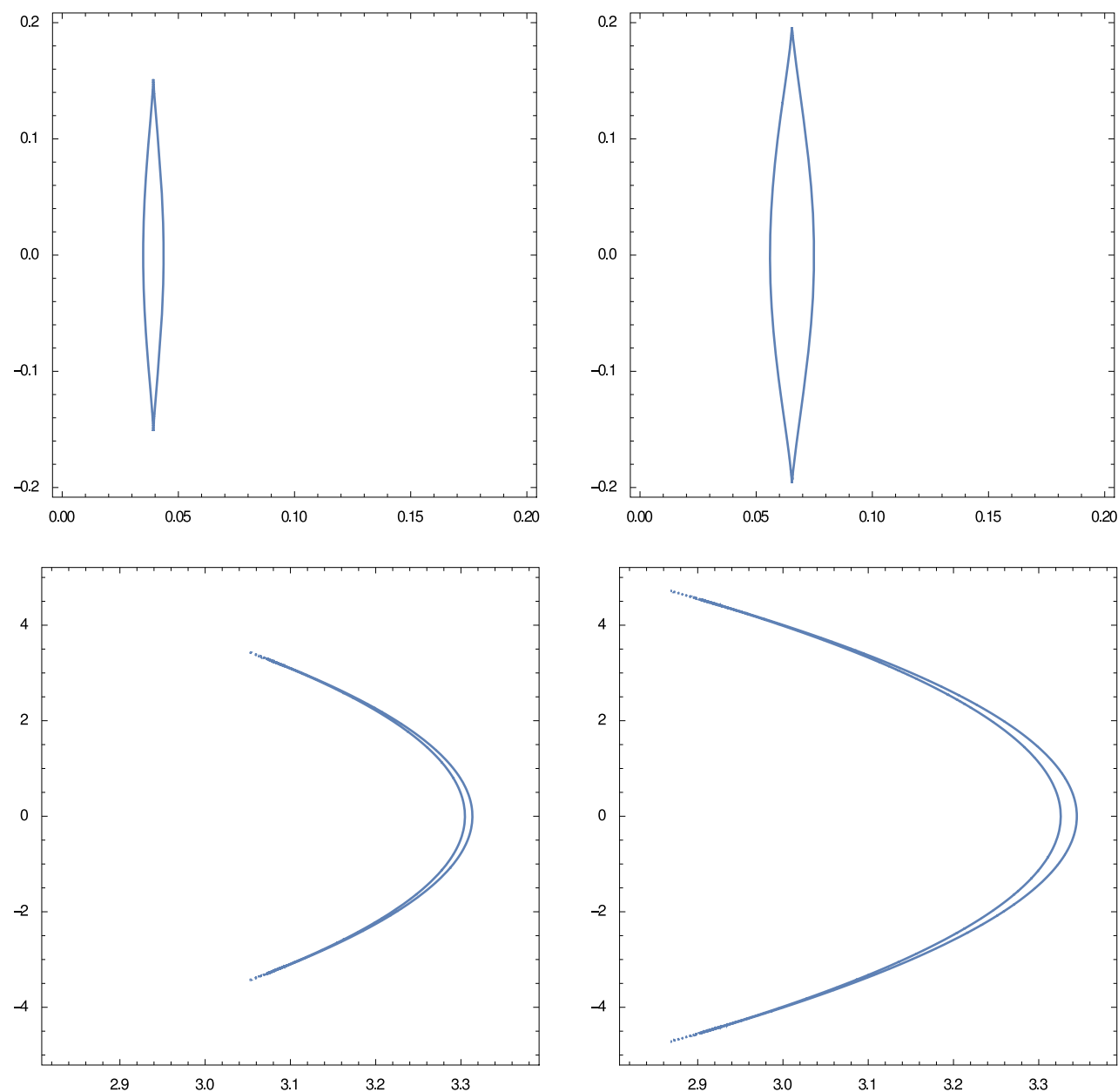

Figure 10. The first two figures show the three-valued region of $\mathrm{dKP}$ at times $\tilde{\tau}=0.03$ and $\tilde{\tau}=0.05$, for $G$ given by $(4.22)$, in the $\left(\xi-\xi_{\mathrm{b}}, \eta-\eta_{\mathrm{b}}\right)$ plane. The second and third figures show the same three-valued region of dKP at the corresponding dKP times $t=t_{\mathrm{b}}(1+\tilde{\tau})^{2}=11.46\left(\tilde{t}=\frac{t-t_{\mathrm{b}}}{t_{\mathrm{b}}}=0.061\right)$ and $t=11.91(\tilde{t}=0.102)$, in the $(x, y)$ plane.

Now we go into the analytic details. For the dKP case:

$$
\tilde{\tau}=\frac{\sqrt{t}-\sqrt{t_{\mathrm{b}}}}{\sqrt{t_{\mathrm{b}}}}
$$

implying

$$
t=t_{\mathrm{b}}(1+\tilde{\tau})^{2}, \quad \tilde{t} \equiv \frac{t-t_{\mathrm{b}}}{t_{\mathrm{b}}}=\tilde{\tau}(2+\tilde{\tau})
$$

If we choose the analytic solution generated by the Gaussian initial condition, with $\epsilon=0.2$, we have seen that wave breaking takes place at $t_{\mathrm{b}}=\tau_{\mathrm{b}}^{2} / 4=10.8$ in the point $\left(x_{\mathrm{b}}, y_{\mathrm{b}}\right)=$ $\left(\xi_{\mathrm{b}}-\tau_{\mathrm{b}}^{2} \eta_{\mathrm{b}}^{2} / 4, \eta_{\mathrm{b}} \tau_{\mathrm{b}}^{2} / 2\right)=(3.27,0)$ of the parabolic wave front $x+y^{2} /(43.2)=\xi_{\mathrm{b}}=3.27$. In this case, if, f.i., $\tilde{\tau}=0.03$, then $t=11.46$ and $\tilde{t}=0.061$. In addition, 


$$
\eta^{\prime}=\frac{y^{\prime}-y_{\mathrm{b}} \tilde{t}}{2 t}, \quad \xi^{\prime}=x^{\prime}+\frac{1}{4 t_{\mathrm{b}}}\left(y^{\prime 2}+2 y_{\mathrm{b}} y^{\prime}-y_{\mathrm{b}}^{2} \tilde{t}\right),
$$

where

$$
x^{\prime}=x-x_{\mathrm{b}}, \quad y^{\prime}=y-y_{\mathrm{b}} .
$$

Using (5.54) and (5.56), the dKP solution reads:

$$
u(x, y, t)=\frac{\epsilon}{\sqrt{t}} G\left(\zeta_{\mathrm{b}}+\zeta^{\prime}, \eta_{\mathrm{b}}+\eta^{\prime}\right)=\frac{\epsilon}{\sqrt{t}}\left(G+G_{\zeta} \zeta^{\prime}+G_{\eta} \frac{y^{\prime}-y_{\mathrm{b}} \tilde{t}}{2 t}+O\left(\frac{\sqrt{t}-\sqrt{t_{\mathrm{b}}}}{\sqrt{t_{\mathrm{b}}}}\right)\right)
$$

where $\zeta^{\prime}$ is the solution of the cubic (5.11) in which $\xi^{\prime}, \eta^{\prime}, \tilde{\tau}$ are replaced by their expressions (5.54), (5.56).

Analogously, the three-valued region in the $(x, y)$ plane, after breaking, is obtained by substituting in (5.32) $\xi^{\prime}, \eta^{\prime}, \tilde{\tau}$ by their expressions (5.54), (5.56).

The obtained equation in the $(x, y, t)$ variables describes a closed curve with two cusps at the points

$$
\vec{x}_{\text {cusp }}^{ \pm}(t)=\left(x_{\text {cusp }}^{ \pm}\left(t^{\prime}\right), y_{\text {cusp }}^{ \pm}(t)\right),
$$

where

$y_{\text {cusp }}^{ \pm}(t)=2\left(\eta_{\mathrm{b}} \pm \sqrt{\frac{2\left|F_{\zeta}\right| F_{\zeta \zeta \zeta}}{\alpha}}\right) t \sqrt{\tilde{\tau}} \sim 2\left(\eta_{\mathrm{b}} \pm \sqrt{\frac{2\left|F_{\zeta}\right| F_{\zeta \zeta \zeta}}{\alpha}}\right) t_{\mathrm{b}} \sqrt{\frac{\sqrt{t}-\sqrt{t_{\mathrm{b}}}}{\sqrt{t_{\mathrm{b}}}}}$

and

$$
\begin{aligned}
& x_{\text {cusp }}^{ \pm}(t) \sim x_{\mathrm{b}}-\frac{1}{4_{\mathrm{b}}}\left[\left(y_{\text {cusp }}^{ \pm}(t)-y_{\mathrm{b}}\right)^{2}+2 y_{\mathrm{b}}\left(y_{\text {cusp }}^{ \pm}(t)-y_{\mathrm{b}}\right)-y_{\mathrm{b}}^{2} \tilde{t}\right] \\
& \quad \pm F_{\eta} \sqrt{\frac{2 F_{\zeta \zeta \zeta}}{\alpha\left|F_{\zeta}\right|} \sqrt{\tau}}+\left(\frac{F}{\left|F_{\zeta}\right|}+\frac{F_{m} F_{\zeta \zeta \zeta}}{\alpha}\right) \tilde{\tau}
\end{aligned}
$$

(see figure 10).

Lastly, the transversal width of this multivalued region is $\Delta_{\text {transv }} \sim\left(y_{\text {cusp }}^{+}(t)-y_{\text {cusp }}^{-}(t)\right)$, while the longitudinal width in the $(x, y)$ plane is the same as in (5.39):

$$
\Delta_{\text {long }} \leqslant \frac{2}{3} \sqrt{\frac{\left|F_{\zeta}\right|}{F_{\zeta \zeta \zeta}}}(\tilde{\tau})^{\frac{3}{2}}
$$

It follows that

$$
\begin{aligned}
& \Delta_{\text {long }}=O\left(\tilde{\tau}^{\frac{3}{2}}\right)=O\left(\left(\frac{\sqrt{t}-\sqrt{t_{\mathrm{b}}}}{\sqrt{t_{\mathrm{b}}}}\right)^{\frac{3}{2}}\right), \\
& \Delta_{\text {transv }}=O\left(\left(t_{\mathrm{b}} \sqrt{\tau}\right)\right)=O\left(t_{\mathrm{b}} \sqrt{\frac{\sqrt{t}-\sqrt{t_{\mathrm{b}}}}{\sqrt{t_{\mathrm{b}}}}}\right), \\
& \Rightarrow \frac{\Delta_{\text {long }}}{\Delta_{\text {transv }}}=O\left(\frac{\tilde{\tau}}{t_{\mathrm{b}}}\right)=O\left(\frac{\tilde{t}}{\sqrt{t_{\mathrm{b}}}\left(\sqrt{t}+\sqrt{t_{\mathrm{b}}}\right)}\right) .
\end{aligned}
$$



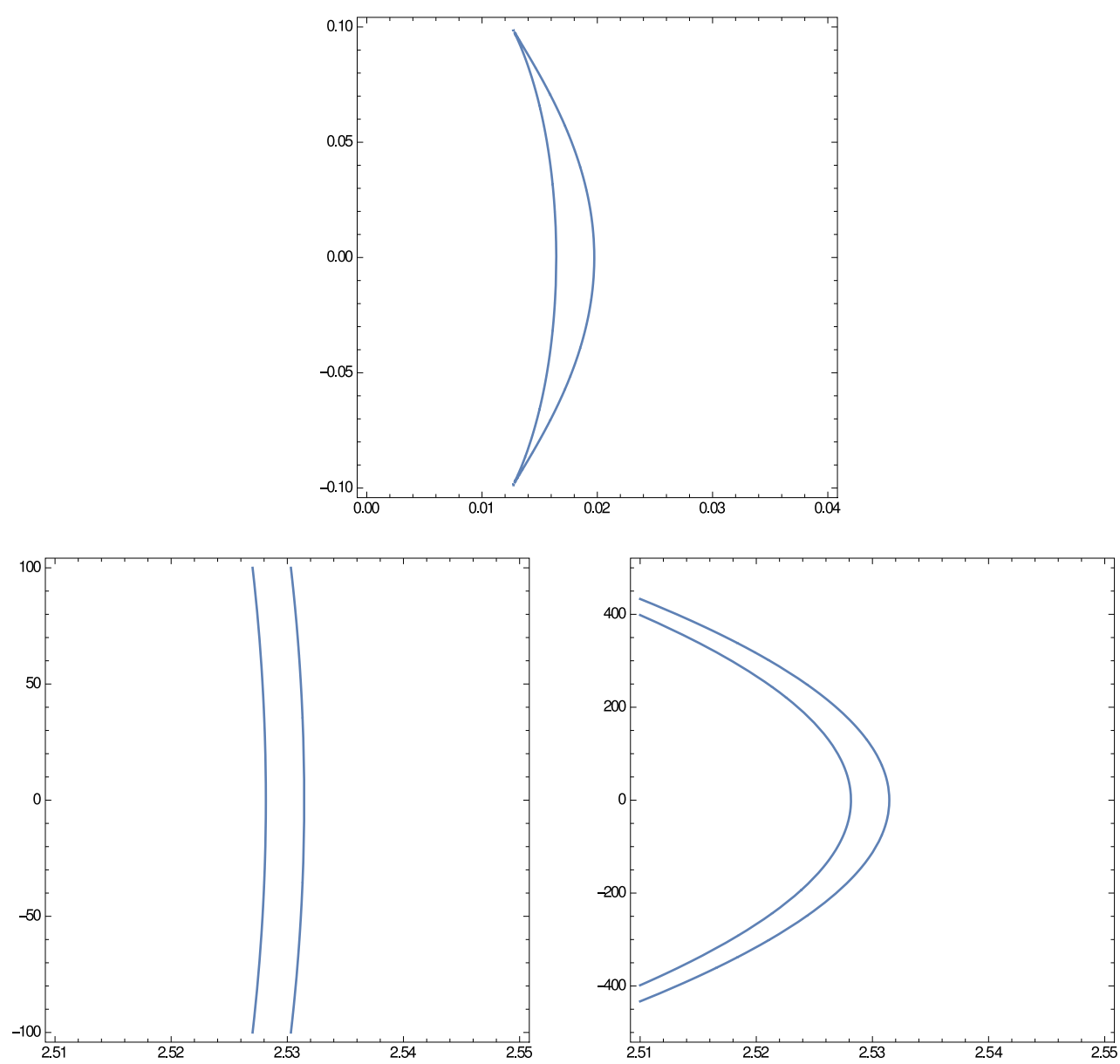

Figure 11. The first figure is the plotting of the three-valued region of mdKP at time $\tilde{\tau}=0.02$, corresponding to $t=t_{\mathrm{b}}^{1.02}=2.19 \times 10^{6}$ and $\frac{t-t_{\mathrm{b}}}{t_{\mathrm{b}}}=0.33$, for $G$ given by (4.22), in the $\left(\xi-\xi_{\mathrm{b}}, \eta-\eta_{\mathrm{b}}\right)$ plane. The second and third figures are different details of the same three-valued region, but in the $(x, y)$ plane of the dKP variables.

For the mdKP case:

$$
\tilde{\tau}=\frac{\ln t-\ln t_{\mathrm{b}}}{\ln t_{\mathrm{b}}},
$$

implying that

$$
t=t_{\mathrm{b}}^{\tilde{\tau}+1}, \quad \tilde{t} \equiv \frac{t-t_{\mathrm{b}}}{t_{\mathrm{b}}}=t_{\mathrm{b}}^{\tilde{\tau}}-1,
$$

and we choose a $\tilde{\tau}$ sufficiently small to have $0<\tilde{t} \ll 1$. For instance, if we choose the analytic solution generated by the Gaussian initial condition, with $\epsilon=0.2$, wave breaking takes place at $t_{\mathrm{b}}=1.64 \cdot 10^{6}$ in the point $\left(x_{\mathrm{b}}, y_{\mathrm{b}}\right)=(2.51,0)$ on the parabola $x+1.52 \cdot 10^{-7} y^{2}=2.51$. In this case, if $\tilde{\tau}=0.02$, then $t=2.18 \times 10^{6}$ and $\tilde{t}=0.33$. 
The mdKP solution reads:

$$
\begin{aligned}
u(x, y, t) & =\frac{\epsilon}{\sqrt{t}} G\left(\zeta_{\mathrm{b}}+\zeta^{\prime}, \eta_{\mathrm{b}}+\eta^{\prime}\right) \\
& =\frac{\epsilon}{\sqrt{t}}\left(G+G_{\zeta} \zeta^{\prime}+G_{\eta} \frac{y^{\prime}-y_{\mathrm{b}} \tilde{t}}{2 t}+O\left(\frac{\ln t-\ln t_{\mathrm{b}}}{\ln t_{\mathrm{b}}}\right)\right)
\end{aligned}
$$

where $\zeta^{\prime}$ is the solution of the cubic (5.11) in which $\xi^{\prime}, \eta^{\prime}, \tilde{\tau}$ are replaced by their expressions (5.64), (5.56).

Analogously, the three-valued region in the $(x, y)$ plane, after breaking, is obtained by substituting in (5.32) $\xi^{\prime}, \eta^{\prime}, \tilde{\tau}$ by their expressions (5.64), (5.56).

The obtained equation in the $(x, y, t)$ variables describes a closed curve with two cusps at the points

$$
\vec{x}_{\text {cusp }}^{ \pm}(t)=\left(x_{\text {cusp }}^{ \pm}\left(t^{\prime}\right), y_{\text {cusp }}^{ \pm}(t)\right)
$$

where

$y_{\text {cusp }}^{ \pm}(t)=2\left(\eta_{\mathrm{b}} \pm \sqrt{\frac{2\left|F_{\zeta}\right| F_{\zeta \zeta \zeta}}{\alpha}}\right) t \sqrt{\tilde{\tau}} \sim 2\left(\eta_{\mathrm{b}} \pm \sqrt{\frac{2\left|F_{\zeta}\right| F_{\zeta \zeta \zeta}}{\alpha}}\right) t \sqrt{\frac{\ln t-\ln t_{\mathrm{b}}}{\ln t_{\mathrm{b}}}}$,

and

$$
\begin{aligned}
& x_{\text {cusp }}^{ \pm}(t) \sim x_{\mathrm{b}}-\frac{1}{4 t_{\mathrm{b}}}\left[\left(y_{\text {cusp }}^{ \pm}(t)-y_{\mathrm{b}}\right)^{2}+2 y_{\mathrm{b}}\left(y_{\text {cusp }}^{ \pm}(t)-y_{\mathrm{b}}\right)-y_{\mathrm{b}}^{2} \tilde{t}\right] \\
& \quad \pm F_{\eta} \sqrt{\frac{2 F_{\zeta \zeta \zeta}}{\alpha\left|F_{\zeta}\right|}} \sqrt{\tilde{\tau}}+\left(\frac{F}{\left|F_{\zeta}\right|}+\frac{F_{\eta \eta} F_{\zeta \zeta \zeta}}{\alpha}\right) \tilde{\tau}
\end{aligned}
$$

(see figure 11).

Lastly, we observe that the longitudinal width of the multivalued region in the $(x, y)$ plane is again the same as in (5.39):

$$
\Delta_{\text {long }} \leqslant \frac{2}{3} \sqrt{\frac{\left|F_{\zeta}\right|}{F_{\zeta \zeta \zeta}}}(\tilde{\tau})^{\frac{3}{2}}=\frac{4 \sqrt{2}}{3} \sqrt{\frac{\left|F_{\zeta}\right|}{F_{\zeta \zeta \zeta}}}\left(\frac{\ln t-\ln t_{\mathrm{b}}}{\ln t_{\mathrm{b}}}\right)^{\frac{3}{2}},
$$

while the transversal width $\Delta_{\text {transv }} \sim\left(y_{\text {cusp }}^{+}(t)-y_{\text {cusp }}^{-}(t)\right)$ becomes very large in a very short time, due to the fact that, when breaking takes place, the parabolic wave front is almost a plane wave front. If, f.i., we are in the explicit case described above, with $\tilde{\tau}=0.02$ and $t_{\mathrm{b}}=1.64 \cdot 10^{6}$ :

$$
\Delta_{\text {transv }}=O(t \sqrt{\tilde{\tau}})=O\left(t_{\mathrm{b}}^{\tilde{\tau}+1} \sqrt{\tilde{\tau}}\right) \sim 8.74 \times 10^{5} .
$$

We end our considerations by remarking that, in the $(x, y)$ plane, the ratio between the longitudinal and transversal widths of the multivalued region is extremely small: $O\left(\tilde{\tau} t_{\mathrm{b}}^{-(\tilde{\tau}+1)}\right) \sim O\left(10^{-8}\right)$; it follows that the multivalued region is essentially one-dimensional (see the second and third figures in figure 11).

\section{Conclusions}

In this paper we have studied what we call the $\operatorname{dKP}(m, n)$ equation (1.1), the natural generalization of the dKP equation (1.3) in $n+1$ dimensions and with nonlinearity of degree $m+1$, a model equation describing the propagation of weakly nonlinear, quasi onedimensional waves in Nature, in the absence of dispersion and dissipation. Apart from the subcases of the Riemann equation (corresponding to $n=1$ ), integrable by the method of 
characteristics, and of the dKP equation (corresponding to $(m, n)=(1,2)$ ), integrable through a novel IST, the $\operatorname{dKP}(m, n)$ equations are not integrable. In spite of that, in this paper we have shown that, if the initial data are localized and small, it is possible to solve analytically the initial value problem for the $\operatorname{dKP}(m, n)$ equation, (i) showing that multidimensional wave breaking takes place only if

$$
1-\frac{m(n-1)}{2} \geqslant 0
$$

and (ii) studying in great analytical detail such wave breaking when it occurs.

An important role in obtaining these results has been played by a family of exact solutions of the $\operatorname{dKP}(m, n)$ equation constructed in this paper, corresponding to its invariance under transformations on the paraboloid

$$
x+\frac{1}{4 t} \sum_{i=1}^{n-1} y_{i}^{2}=\text { constant }
$$

These exact solutions, describing waves constant on their paraboloidal wave front and breaking simultaneously on it, appear, together with the longtime behavior of the linearized theory, to be the basic ingredients in the description of the asymptotic nonlinear regime of the above Cauchy problem.

\section{References}

[1] Santucci F 2012 Onde debolmente non lineari e quasi unidimensionali in natura. La rottura di tali onde e il problema della regolarizzazione dissipativa Tesi della Laurea Specialistica in Fisica (La Sapienza: Dipartimento di Fisica dell'Università di Roma) (Academic year 2011-2012, supervisor P M Santini) pp 177-286

[2] Manakov S V and Santini P M 2011 On the dispersionless Kadomtsev-Petviashvili equation in $n+1$ dimensions: exact solutions, the Cauchy problem for small initial data and wave breaking J. Phys. A: Math. Theor. 44405203

[3] Whitham G B 1974 Linear and Nonlinear Waves (New York: Wiley)

[4] Lin C, Reissner E and Tien H S 1948 On two-dimensional nonsteady motion of a slender body in a compressible fluid J. Math. Phys. 27 220-31

[5] Timman R 1962 Unsteady motion in transonic flow Symp. Transsonicum Aachen ed K Oswatitsch (Berlin: Springer) pp 394-401

[6] Zobolotskaya E A and Khokhlov R V 1969 Quasi-plane waves in the nonlinear acoustics of confined beams Sov. Phys. - Acoust. 15 N.1

[7] Kadomtsev B B and Petviashvili V I 1970 On the stability of solitary waves in weakly dispersing media Sov. Phys. - Dokl. 15 N.6

[8] Wang X P, Ablowitz M J and Segur H 1994 Wave collapse and instability of solitary waves of a generalized Kadomtsev-Petviashvili equation Physica D 78 241-65

[9] Ablowitz M J and Segur H 1981 Solitons and the Inverse Scattering Transform (Philadelphia, PA: SIAM)

[10] Ablowitz M J and Clarkson P A 1991 Solitons, Nonlinear Evolution Equations and Inverse Scattering (Cambridge University Press)

[11] Turitsyn S and Falkovitch G 1985 Stability of magneto-elastic solitons and self-focusing of sound in antiferromagnets Sov. Phys. - JETP 62 146-52

[12] Zakharov V E and Shabat A B 1974 A scheme for integrating the nonlinear equations of mathematical physics by the method of the inverse scattering problem Funct. Anal. Appl. 8 $226-35$

[13] Dryuma V S Analytic solution of the two-dimensional Korteweg-de Vries equation Sov. Phys. JETP Lett. 19 381-8

[14] Manakov S V 1981 The inverse scattering transform for the time dependent Schrödinger equation and Kadomtsev-Petviashvili equation Physica 3D 420-7 
[15] Ablowitz M J, BarYaacov D and Fokas A S 1983 On the inverse scattering transform for the Kadomtsev-Petviashvili equation Stud. Appl. Math. 69 135-42

[16] Novikov S, Manakov S V, Pitaevskii L P and Zakharov V E 1984 Theory of Solitons (Monographs in Contemporary Mathematics) (Springer: New York)

[17] Manakov S V, Santini P M and Takhtajan L A 1980 Asymptotic behaviour of the solutions of the Kadomtsev-Petviashvili equation (two dimensional Korteweg-de Vries equation) Phys. Lett. A $\mathbf{7 5} 451$

[18] Anders I A, Kotlyarov V P and Khruslov E Y 1994 Curved asymptotic solutions of the Kadomtsev-Petviashvili equation Theor. Math. Phys. 99 402-8

[19] Kiselev O M 2001 Asymptotics of a Solution of the Kadomtsev-Petviashvili-2 Equation, Proc. Steklov Institute of Mathematics (supplementary issue) suppl 1 S107-30

[20] Kiselev O M Asymptotic behaviour of a solution for Kadomtsev-Petviashvili-2 equation (arXiv: math-ph/0003014)

[21] Manakov S V and Santini P M Inverse scattering problem for vector fields and the heavenly equation (arXiv:nlin.SI/0512043)

[22] Manakov S V and Santini P M 2006 Inverse scattering problem for vector fields and the Cauchy problem for the heavenly equation Phys. Lett. A $359613-9$

[23] Manakov S V and Santini P M 2006 The Cauchy problem on the plane for the dispersionless Kadomtsev-Petviashvili equation JETP Lett. 83 462-6

[24] Grinevich P G, Santini P M and Wu D 2015 The Cauchy problem for the Pavlov equation Nonlinearity 28 3709-54

[25] Pavlov M V 2003 Integrable hydrodynamic chains J. Math. Phys. 44 4134-56

[26] Manakov S V and Santini P M 2008 On the solutions of the dKP equation: nonlinear Riemann Hilbert problem, longtime behaviour, implicit solutions and wave breaking J. Phys. A: Math. Theor. 41055204

[27] Manakov S V and Santini P M 2012 On the wave breaking of solutions of the dispersionless Kadomtsev-Petviashvili equation at finite time Teor. Mat. Fiz. 172 1118-26

[28] Grava T, Klein C and Egger J 2016 Shock formation in the dispersionless KadomtsevPetviashvili equation Nonlinearity 29 1384-416

[29] Dubrovin B, Grava T and Klein C 2016 On the critical behaviour in generalized KadomtsevPetviashvili equation Physica D 333 157-70

[30] Kusher A, Lychagin V and Rubtsov V 2007 Contact Geometry and Non-Linear Differential Equations (Cambridge: Cambridge University Press)

[31] Manakov S V and Santini P M 2011 Solvable vector nonlinear Riemann problems, exact implicit solutions of dispersionless PDEs and wave breaking J. Phys. A: Math. Theor. 44345203

[32] Abramowitz M and Stegun I A (ed) 1972 Handbook of Mathematical Functions with Formulas, Graphs, and Mathematical Tables (New York: Dover)

[33] Erdelyi A, Magnus W, Oberhettinger F and Tricomi F G 1953 Higher Transcendental Functions vol 1 (McGraw-Hill) 